University of Wollongong

Research Online

Faculty of Engineering and Information

Faculty of Engineering and Information

Sciences - Papers: Part B

Sciences

2017

Experimental and numerical investigations into hydraulic behaviour of coir fibre drain

Thanh Trung Nguyen

University of Wollongong, ttnguyen@uow.edu.au

Buddhima Indraratna

University of Wollongong, indra@uow.edu.au

Follow this and additional works at: https://ro.uow.edu.au/eispapers1

Part of the Engineering Commons, and the Science and Technology Studies Commons

Research Online is the open access institutional repository for the University of Wollongong. For further information contact the UOW Library: research-pubs@uow.edu.au 


\title{
Experimental and numerical investigations into hydraulic behaviour of coir fibre drain
}

\begin{abstract}
Over many decades, natural fibre bundles have been widely used for drainage and filtration applications because of their favourable hydraulic conductivity and abundance in Asian countries. In recent times, natural (biodegradable) coir and jute drains, which are environmentally friendly, have been considered in lieu of conventional geosynthetic wick drains for soft clay consolidation in Australian coastal regions. However, there is a lack of a computational framework to predict the hydraulic behaviour of fibre drains on the basis of micromechanical (fabric) characteristics. Employing computational fluid dynamics (CFD) coupled with the discrete element method (DEM) to model the hydraulic behaviour of fibrous materials has shown promise in an earlier 2016 study by Nguyen and Indraratna, which considered an idealized parallel arrangement of fibres for simplicity. This paper aims to broaden the application of the coupled CFD-DEM technique to real fibres (coconut coir) considering both nontwisted and twisted fibre bundles that have more complex porous structure. The hydraulic conductivity determined from the numerical approach is validated with the experimental results, and also compared with the analytical prediction based on the conventional Kozeny-Carmen (KC) approach. The current study shows that the CFD-DEM technique can capture well the fluid flow characteristics of a nonuniform fibrous structure, including dense twisted coir bundles.

\section{Disciplines}

Engineering | Science and Technology Studies
\end{abstract}

\section{Publication Details}

Nguyen, T. Trung. \& Indraratna, B. (2017). Experimental and numerical investigations into hydraulic behaviour of coir fibre drain. Canadian Geotechnical Journal, 54 (1), 75-87. 


\title{
EXPERIMENTAL AND NUMERICAL INVESTIGATIONS INTO HYDRAULIC BEHAVIOUR OF COIR FIBRE DRAIN
}

\author{
Thanh Trung Nguyen \\ PhD student, \\ Centre for Geomechanics and Railway Engineering, University of Wollongong, \\ Wollongong City, NSW 2522, Australia

\section{Buddhima Indraratna ${ }^{1}$} \\ BSc (Hons., Lond.), MSc (Lond.), DIC, PhD (Alberta), FTSE, FIEAust., FASCE, FGS \\ Distinguished Professor of Civil Engineering, \\ Faculty of Engineering and Information Sciences, \\ Director, Centre for Geomechanics and Railway Engineering, University of \\ Wollongong, Wollongong City, NSW 2522, Australia
}

Words: 6482

Figures: 13

Tables: 02

Submitted to: Canadian Geotechnical Journal

${ }^{1}$ Corresponding author: Buddhima Indraratna (e-mail: indra@uow.edu.au) 


\section{Abstract}

\section{EXPERIMENTAL AND NUMERICAL INVESTIGATIONS INTO} HYDRAULIC BEHAVIOUR OF COIR FIBRE DRAIN

Over many decades, natural fibre bundles have been used widely for drainage and

filtration applications because of their favourable hydraulic conductivity and abundance in Asian countries. In recent times, natural (biodegradable) coir and jute drains that are environmentally-friendly have been considered in lieu of conventional geosynthetic wick drains for soft clay consolidation in Australian coastal regions. However there is a lack of computational framework to predict the hydraulic behaviour of fibre drains on the basis of micromechanical (fabric) characteristics. Employing the Computational Fluid Dynamics (CFD) coupled with the Discrete Element Method (DEM) to model the hydraulic behaviour of fibrous materials has shown promise in an earlier study (Nguyen and Indraratna 2016), which considered an idealised parallel arrangement of fibres for simplicity. This paper aims to broaden the application of the coupled CFD-DEM technique to real fibres (coconut coir) considering both non-twisted and twisted fibre bundles that have more complex porous structure. The hydraulic conductivity determined from the numerical approach is validated with the experimental results, and also compared to the analytical prediction based on the conventional Kozeny-Carmen $(\mathrm{KC})$ approach. The current study shows that the CFD-DEM technique can capture well the fluid flow characteristics of a non-uniform fibrous structure, including dense twisted coir bundles.

Keywords: geomaterials; natural fibre drains; hydraulic conductivity; fluid-particle coupling; Kozeny-Carmen approach. 
Natural fibres drains which are emerging as environmentally-friendly alternative to

27 the conventional geosynthetic materials have received increasing attention in the recent years, 28 including their application as prefabricated vertical drains (PVD) in soft soil consolidation for 29 infrastructure development (Lee et al. 1994; Venkatappa Rao et al. 2000; Kim and Cho 2009). As the process of biodegradation of these natural materials takes several years (i.e. after serving the period of primary consolidation), PVDs made from jute and coir are now becoming increasingly popular, and manufactured for worldwide export in South and Southeast Asian countries. Most applications where naturally occurring materials are used for either filtration or drainage in geotechnical practice are based on their favourable hydraulic conductivity (Kim et al. 2001; Asha and Mandal 2012). Consequently, there is a need for creating a computational scheme which can predict the hydraulic behaviour of these fibrous media accurately. Nguyen and Indraratna (2016) used a combined approach between the Discrete Element Method (DEM) and Computer Fluid Dynamics (CFD) to model fibrous geomaterials, and found a good agreement between this solution and previous studies in relation to fluid flow characteristics. Although this study verified the CFD-DEM technique by only applying it to predict the permeability of an idealised arrangement of parallel fibres, it provided a launching pad to extend the numerical approach further to encompass real natural fibres that have a more complex porous structure (e.g., twisted fabric). Many previous studies clarified the hydraulic behaviour of natural fibres on a macro scale; for instance, Venkatappa Rao et al. (2000), and Asha and Mandal (2012) studied the discharge capacity of jute drains, while Asha et al. (2012) studied the transverse permeability 47 of jute geotextiles. However, there is a distinct lack of studies focused on micro 48 characteristics of porous media that could provide a better insight into the hydraulic 
behaviour of natural fibre drains. Since the permeability of porous materials depends on the size and arrangement of fibres (Ozgumus et al. 2014), variations in the micro-structure of fibrous media can significantly change the hydraulic conductivity of the whole drain system and therefore the overarching objective of this study.

The Kozeny-Carmen (KC) method is usually preferred in practice due to its computational convenience. Previous studies (Sullivan 1942; Gutowski et al. 1987; Gebart 1992) have shown that the KC concept is also applied for longitudinal flow through fibrous media, where the relationship between permeability and the porosity is given by:

$$
K=\frac{D_{f}^{2} n^{3}}{16 k_{k}(1-n)^{2}}
$$

where $D_{f}$ is the average diameter of fibres in the drain, $n$ is the porosity (or void fraction) of the medium, and $k_{k}$ is the Kozeny constant that is usually determined by experimental methods. This empirical constant has a wide range of values that depend on the porous characteristics of media, as summarised by Ozgumus et al. (2014), which is why specifying $k_{k}$ for practical applications is still being debated.

This study focuses on the hydraulic behaviour of coir (coconut) fibre, because it is one of the most commonly used natural fibres worldwide. Primary tests such as tension and bending were carried out on coir fibre to determine its basic mechanical properties, and an experimental scheme to determine the hydraulic conductivity of the coir bundles with different porous features was then carried out. The experimental results were then used to

67 validate the CFD-DEM technique and also compared with the conventional $\mathrm{KC}$ analytical 68 approach. 


\section{Experimental investigation on the longitudinal permeability of coir fibres}

\section{Fibrous drain}

A natural fibre drain is a combination of single fibres in a certain arrangement that enables fluid to flow through its porous medium. There are currently a variety of fibre drains made from natural fibres such as jute, coir, and straw with either circular or band shaped cross-sections. Of these, coir and jute are the most preferable due to their abundance in developing nations in South and Southeast Asia. Coir fibre consists of approximately $40-45 \%$ lignin and 35-45\% cellulose components (Gupta 2011) that make this fibre more robust and durable than jute which only has around 12\% lignin (Som et al. 2009). In this study, dry brown coir fibres provided by the National Jute Board of India (NJBI) were used to generate fibre drains which were then subjected to an experimental investigation into their hydraulic behaviour. Physical properties of the coir fibres which were obtained by carrying out laboratory tests (represented in the following parts of this paper) are summarized in Table 1. Note that to minimize the influence that the water absorption of coir fibres could have on the hydraulic test, coir fibre drains were soaked in water to make them saturated before testing. Straight and undamaged coir fibres were used to create fibre drains, of which two types of fibre structure were investigated, non-twisted and twisted bundles. The non-twisted bundles consisted of individual coir fibres arranged almost in parallel (no twisting) whereas in the twisted structure, single fibres were packed together and twisted around the longitudinal axis of the bundle to a certain degree (Fig. 1a); fundamentally, the tighter the fibres were twisted together, the denser and stronger their composition. The fibre fraction was approximately manipulated by the number of single fibres in the bundle, such that as more fibres are packed, the denser the bundle generated. Fig. $1 \mathrm{~b}$ and $1 \mathrm{c}$ show how non-twisted and twisted bundles can be generated using coir fibres, respectively. 


\section{Experiment to determine the hydraulic conductivity of a fibre drain}

Experimental methods with unidirectional and radial flow are the two common approaches used to determine the hydraulic conductivity of fibrous materials (Sharma and Siginer 2010). Fibres are pre-formed in a mould before being subjected to fluid flow, and low injection pressure is required to ensure the fibres remain in a static condition. Either the fluid velocity or pressure is controlled while the fluid is flowing. In this study, an experimental process based on the pressure controlled model was designed and established (Fig. 2a).

The bundles of fibre which had an identical cross sectional area and length were placed inside $4 \mathrm{~mm}$ diameter by $100 \mathrm{~m}$ long hard tubes (Fig. $2 \mathrm{~b}$ and $2 \mathrm{c}$ ). The interior surface of the tube was smooth to reduce the effect of friction on fluid flow. The inlet of the tube was connected to a constant head water tank with adjustable elevation to generate water flow under different static pressures, and manometers were installed to measure the water heads at the inlet and outlet of the tube. Water from the inlet drained through the fibrous environment was collected at the outlet of the tube, so that the hydraulic gradient of the flow could be obtained with respect to the length and the difference in water heads between the inlet and outlet of the bundle. The volume of water discharged through the fibrous system over time was recorded. The viscosity of the water was determined according to its temperature measured, in accordance with ASTM D4716 (ASTM 2008). The dynamic viscosity of the water used in this study was $1.004 \times 10^{-3} \mathrm{~Pa} . \mathrm{s}$ at $20^{\circ} \mathrm{C}$.

To obtain the porous characteristics of this fibrous system, a series of micro-analyses were carried out in the following manner: after the hydraulic test, the tube containing fibres was dried by blowing warm dry air through the tube, which was then immersed into a mixture of resin and hardener to maintain the structure of the fibrous medium. These samples were then subjected to a stacking technique along the fibres in which a series of photos of the cross-section of the bundle were taken along the longitudinal axis of samples with an optical 
118 microscope. Image analysis techniques that are available in the ImageJ software (Rasband

119 2014) were carried out on these photos to obtain geometrical information such as the cross

120 sectional area and the coordinates of individual fibres in a given drain to enable the fibrous

121 system to be reconstructed on the DEM framework. Fig. 3a shows an example of cross-

122 sections of coir fibres under microscopic observation. The coir fibres have almost round

123 cross-sections that are reasonable to be modelled by spherical particles which are commonly used in DEM.

Fig. $3 b$ shows how the diameters of the coir fibres are distributed within a typical

126 drain with the most common sizes being from 150 to $300 \mu \mathrm{m}$, and which accounted for more

127 than $70 \%$ of the total number of fibres. The average diameter of the coir in this study was 128 approximately $236 \mu \mathrm{m}$, which is within the common range of this fibre (100 to $530 \mu \mathrm{m})$, as

129 summarized by Ali (2010). The density of the fibre investigated in this study was $1160 \mathrm{~kg} / \mathrm{m}^{3}$

130 that corroborates with those reported by other studies (Rao and Rao 2007; Defoirdt et al. 131 2010). The water content of the fibre (Table 1) was determined as the ratio of the mass of water in the fibre to the mass of dried fibre. time $t$, the hydraulic conductivity $k$ was estimated as follows:

$$
k=\frac{V_{\mathrm{f}} R_{t}}{i A_{t} t}
$$

135 where $R_{\mathrm{t}}$ is the correction factor for the viscosity of water, depending on the water temperature (ASTM 2008); $A_{t}$ is the internal cross sectional area of the tube and $i$ is the

137 hydraulic gradient. Note that in this experiment, fluid flow was investigated under a small

138 difference in water heads to ensure laminar flow, while the discharge volume $V_{\mathrm{f}}$ was 139 measured at a steady state of flow. 
142 Element Method (DEM) code onto an open source framework called LIGGGHTS (Kloss and

143 Goniva 2010), while fluid behaviour is described by the Computational Fluid Dynamics

144 (CFD). Interaction between the fluid-solid phases is carried out by a mutual platform called

145 CFDEM (Goniva et al. 2010) which exchanges and updates information between each phase.

146 The behaviour of particles and fluid is described on the basis of the following concepts.

\section{Fluid behaviour}

This study assumed an incompressible fluid with conservation of mass where the fluid variables, including the velocity $U_{f}$ and pressure $p$ are governed in individual cells as represented by the following Navier-Stokes equations.

$$
\frac{\partial n}{\partial t}+\nabla \cdot\left(n U_{f}\right)=0
$$

$$
\frac{\partial\left(\rho_{f} n U_{f}\right)}{\partial t}+\nabla \cdot\left(\rho_{f} n U_{f} U_{f}\right)=-n \nabla p-f_{p}+\nabla \cdot(n \tau)+n \rho_{f} g
$$
while. The porosity $n$ of a certain fluid cell is defined as the ratio of the void volume in a cell to its total volume: $n=V_{v} / V_{c}=1-V_{p} / V_{c}$ where $V_{v}, V_{p}$ are the volumes of the void and particles occupied in a cell, respectively; and $V_{c}$ is the volume of the cell. $f_{p}$ is the mean volumetric particle-fluid interaction force representing the effect of the solid phase on the fluid phase within the cell. By considering that the fluid cell $\alpha$ contains $n_{p}$ particles, $f_{p}$ of cell $\alpha$ can be

157 estimated by Equation (5) as follows: 


$$
f_{p, \alpha}=\sum_{i=1}^{n_{p, \alpha}} \varpi_{i \alpha}\left(\frac{F_{p, i}}{V_{c, \alpha}}\right)
$$

158 where $F_{p, i}$ is the total force acting on particle $i ; V_{c, \alpha}$ is the volume of the fluid cell $\alpha$. The

159 factor $\varpi_{i \alpha}$ representing the volumetric portion of particle $i$ residing in cell $\alpha$ is estimated as

160 the ratio of the exact volumetric portion of particle $i$ in cell $\alpha$ to the total volume of cell $\alpha$.

161 The viscous stress tensor $\tau$ can be written in relation to the fluid viscosity $\mu_{f}$ and 162 velocity $U_{f}$ as follows:

$$
\tau=\mu_{f}\left[\left(\nabla U_{f}\right)+\left(\nabla U_{f}\right)^{T}\right]
$$

\section{Particle behaviour} components, is governed by the following equations:

$$
m_{i} \frac{d U_{p, i}}{d t}=\sum_{j=1}^{n_{i}^{c}} F_{c, i j}+F_{f, i}+F_{g, i}
$$

$$
I_{i} \frac{\omega_{p, i}}{d t}=\sum_{j=1}^{n_{i}^{c}} M_{c, i j}
$$

where $U_{p, i}$ and $\omega_{p, i}$ are the translational and angular velocities of particle $i$, respectively; $m_{i}$ is

167 the mass and $I_{i}$ is referred to as the inertia moment of particle $i ; F_{c, i j}$ and $M_{c, i j}$ are the contact 168 force and torque acting on particle $i$ by particle $j$ (or walls), while $n_{i}^{c}$ is the number of total contacts of particle $i . F_{g, i}$ is referred to as the gravitational force, while $F_{f, i}$ is the total fluid-

170 particle interaction force imported from the fluid domain acting on particle $i$.

172 particle motion, can consist of the drag force, the pressure gradient force, the viscous force,

173 and other unsteady forces such as the virtual mass, the Basset and the lift forces (Zhu et al. 
174 2007; Zhou et al. 2010). Previous studies (Zhu et al. 2007; Zhou et al. 2010) have shown that any unsteady forces are usually insignificant compared to the drag and pressure gradient

176 forces, especially in laminar flow. Assuming laminar flow in this study, the unsteady forces 177 were ignored.

178 The drag force acting on particle $i$ positioning in fluid cell $\alpha$, according to De Felice's 179 solution which was used and verified by Zhou et al. (2010), is given by:

$$
F_{d, i}=\frac{1}{8} C_{d, \alpha} \rho_{f} \pi D_{p, i}^{2} n_{\alpha}^{2}\left(\mathrm{U}_{f, \alpha}-\mathrm{U}_{p, i}\right)\left|\mathrm{U}_{f, \alpha}-\mathrm{U}_{p, i}\right| n_{\alpha}{ }^{-\chi}
$$

180 where $U_{f, \alpha}$ and $U_{p, i}$ are referred to as the averaged velocity of fluid in cell $\alpha$ and the velocity 181 of particle $i$ residing in cell $\alpha$, respectively; $D_{p, i}$ is the diameter of particle $i$ where the drag 182 force acts on, and $C_{d, i}$ is the fluid-particle drag coefficient which is calculated by:

$$
C_{d, i}=\left(0.63+\frac{4.8}{\sqrt{R e_{p, i}}}\right)^{2}
$$

183 where $R e_{p, i}$ is the particle Reynolds number which is determined by:

$$
R e_{p, i}=\frac{n_{\alpha} \rho_{f} D_{p, i}\left|U_{f, \alpha}-U_{p, i}\right|}{\mu_{f}}
$$

184 In Equation (9) the porosity function $n_{\alpha}^{-\chi}$ represents the presence of other particles in cell $\alpha$

185 in relation to the power factor $\chi$ which is a function of the Reynolds number $R e_{p, i}$ and is 186 estimated by:

$$
\chi=3.7-0.65 \exp \left[-\frac{\left(1.5-\log _{10} R e_{p, i}\right)^{2}}{2}\right]
$$

187 Note that according to Equation 9, the drag force depends mainly on the difference between 188 the velocities of fluid $U_{f, \alpha}$ and particle $U_{p, i}$. For dense and reinforced conditions where 
189 particles are strictly confined, the drag force becomes more significant and depends mainly

190 on the fluid velocity.

191 The force $F_{h, i}$ accounting for the stress gradient of fluid imparted onto particle $i$

192 having a volume $V_{p, i}$ can be written by (Kafui et al. 2002):

$$
F_{h, i}=(-\nabla p+\nabla . \tau) V_{p, i}
$$

193 where $p$ and $\tau$ are the pressure and viscous stress tensors constituting the average stress

194 tensor $\xi_{f}$ of fluid that can be given by:

$$
\xi_{f}=-p \delta+\tau
$$

195 where $\delta$ is the identity (unit) tensor. The pressure gradient force generated due to the

196 difference in fluid pressure acting on particle $i$ can be separated into two parts: $(i)$ the

197 buoyancy force $F_{b, i}$, and (ii) the component $F_{\nabla p, i}$ generated by the acceleration pressure

198 gradient which represents the pressure difference of fluid flowing over a surface, resulting in

199 an acceleration (Newton's second law). These components can be computed individually, as

200 shown below:

$$
\begin{aligned}
& F_{\nabla p, i}=-(\nabla p) V_{p, i} \\
& F_{b, i}=-\rho_{f} g V_{p, i}
\end{aligned}
$$

201 In this study, the fluid-particle coupling technique was applied to model the hydraulic

202 behaviour of coir fibres that were packed in various porous structures, which can result in a

203 deviation of fluid velocity distributed over the fibre domain. The viscous force should 204 therefore be considered as shown below: 


$$
F_{\nabla . \tau, i}=(\nabla . \tau) V_{p, i}
$$

206 can be represented as:

$$
F_{f, i}=F_{d, i}+F_{\nabla p, i}+F_{b, i}+F_{\nabla . \tau, i}
$$

\section{Modelling fibres in DEM}

The Parallel Bond Model (PBM) in DEM has proven to be reasonable when

209 simulating a linear stress-strain behaviour and brittle fracture (Cho et al. 2007; Lisjak and

210 Grasselli 2014). Nguyen and Indraratna (2016) have also shown that the PBM can be applied

211 well to capture the tension behaviour of jute and bamboo. However the tension test

212 (represented in the following parts of this paper) carried out on the coir fibre in this study (see

213 Fig. 4) revealed that the fibre had a non-linear stress-strain curve that requires the PBM to be 214 modified accordingly.

215 A coefficient $\beta_{b}$ including shear and normal components, $\beta_{b n}$ and $\beta_{b s}$, which enable 216 the bond model to capture various orders of displacements of particles in bonding are 217 introduced to the conventional PBM proposed by Potyondy and Cundall (2004). The 218 behaviour of particle bonding in DEM, including the tension, shear, and bending components 219 are then governed by the following equations:

$$
\begin{aligned}
& \Delta F_{b n, i j}=-k_{b n}\left(\Delta D_{n}\right)^{\beta_{b n}} A_{b} \\
& \Delta F_{b s, i j}=-k_{b s}\left(\Delta D_{s}\right)^{\beta_{b s}} A_{b} \\
& \Delta M_{b n, i j}=k_{b s} J_{b} \Delta \theta_{n}
\end{aligned}
$$




$$
\Delta M_{b s, i j}=k_{b n} I_{b} \Delta \theta_{s}
$$

220 In the above, the subscripts $n$ and $s$ indicate the normal and shear components, respectively; $221 \Delta F, \Delta M$ are increments of the bond force $F$ and moment $M$, respectively, generated due to 222 the relative translational and rotational displacements $\Delta D_{n}, \Delta D_{s}, \Delta \theta_{n}, \Delta \theta_{s}$ between two 223 particles in the bond; $k_{b}$ is the bond stiffness. $A_{b}, I_{b}$ and $J_{b}$ are the area, moment of inertia, and 224 the polar moment of inertia of the bond cross-section, respectively. These parameters are 225 given by:

$$
\begin{aligned}
& A_{b}=\pi R_{b}^{2} \\
& I_{b}=\frac{1}{4} \pi R_{b}^{4} \\
& J_{b}=\frac{1}{2} \pi R_{b}^{4}
\end{aligned}
$$

226 In the above, $R_{b}$ is the radius of the bond region determined by:

$$
R_{b}=\lambda \min \left(R_{i}, R_{j}\right)
$$

where $R_{i}$ and $R_{j}$ are the radius of particles $i$ and $j$ in bonding; $\lambda$ is the bond radius multiplier to optimise the virtual sharing area between two particles. The maximum normal and shear stresses acting on the bond periphery are computed as follows:

$$
\begin{gathered}
\sigma_{\text {max }}=\frac{-F_{b n, i j}}{A}+\frac{\left|M_{\mathrm{bs}, i j}\right|}{I} R_{b} \\
\tau_{\max }=\frac{\left|F_{\mathrm{bs}, i j}\right|}{A}+\frac{\left|M_{\mathrm{bn}, i j}\right|}{J} R_{b}
\end{gathered}
$$

230 The bond is broken when either $\sigma_{\max }$ or $\tau_{\max }$ exceeds its corresponding strength $\sigma_{b}$ and $\tau_{b}$.

231 These parameters combined with $k_{b, n}, k_{b, s}, \beta_{b n}$ and $\beta_{b s}$ can be determined by primary tests

232 such as testing the tension of real fibres. If the two components of the coefficient $\beta_{b}$ in 
equations (19) and (20) are both equal to 1, then the conventional PBM is achieved. The radius of fibre particles in DEM was estimated based on the size of real fibres obtained from 235 micro-analyses.

237 equations:

$$
\Delta t_{h}=2.87\left(\frac{m_{e}^{2}}{R_{e} Y_{e}^{2} U_{p, \max }}\right)^{0.2}
$$

$$
\Delta t_{r}=\frac{\pi R}{(0.1631 \times v+0.8766)} \sqrt{\frac{\rho_{p}}{G}}
$$

238 where $v$ is Poisson's ratio; $\rho_{\mathrm{p}}$ is the density and $G$ is the shear modulus of the particle; $R$ 239 denotes the average radius of particles and $U_{p \text {, } \max }$ is the maximum relative velocity of 240 particles. $R_{e}, Y_{e}$ and $m_{\mathrm{e}}$ are the effective radii, Young's modulus, and the mass of particles $i$ 241 and $j$ in contact, respectively, and are computed as follows:

$$
\begin{aligned}
& \frac{1}{R_{e}}=\frac{1}{R_{i}}+\frac{1}{R_{j}} \\
& \frac{1}{Y_{e}}=\frac{1-v_{i}^{2}}{Y_{i}}+\frac{1-v_{j}^{2}}{Y_{j}} \\
& \frac{1}{m_{e}}=\frac{1}{m_{i}}+\frac{1}{m_{j}}
\end{aligned}
$$

For the coir fibres with a minimum diameter of $102 \mu \mathrm{m}$ and an average density of

$2431160 \mathrm{~kg} / \mathrm{m}^{3}$ used in this study, the time step of the DEM $\Delta t_{p}=1 \times 10^{-8}$ was found suitable for 244 computation.

Contact between the fibre particles in DEM can be depicted on the basis of the spring246 dashpot models, by adopting either linear or nonlinear spring and damping coefficients 
247 (Kloss et al. 2012). The reaction forces, including the normal and shear components of two 248 particles in contact, are dependent on the overlap of particles (Cundall and Strack 1979). Note

249 that while the equivalent volume based diameter was considered for the porosity of fluid 250 cells, the real diameter of the fibres was used to capture the contact between particles, as 251 proposed by Nguyen and Indraratna (2016).

\section{Calibrate the parameters of the fibre bond model}

To obtain parameters for particle bonding, primary tension and bending tests were carried out on single fibres. Tension tests with a constant strain rate of $3 \mathrm{~mm} / \mathrm{min}$ were carried out on 22 individual coir fibres which had their diameters measured in advance under optical microscope. Note that the number of fibres selected for testing was made with respect to previous studies (Defoirdt et al. 2010; Biswas et al. 2013) which have shown an acceptable accuracy of tension tests on single fibres. The tension force and displacement were recorded over time until the coir fibre broke. The averaged result from the tension test showing the non-linear stress-strain relationship of coir is shown in Fig. 4 where the curve indicates a brittle fracture of the coir fibres under tension. The average tensile strength of coir fibre obtained in this study (i.e., $\sigma_{b n}=188 \mathrm{MPa}$ with a standard deviation of $34 \mathrm{Mpa}$ ) corroborates with the values reported in a previous study by Defoirdt et al. (2010), which found the tensile strength of brown coir within a wide range, i.e., from 186 to $343 \mathrm{MPa}$. The components $k_{b n}$ and $\beta_{b n}$ in the numerical simulation were obtained on the basis of the experimental stressstrain curve. Fig. $4 \mathrm{c}$ shows how well the modified PBM with $k_{b n}=1.01 \times 10^{11} \mathrm{~Pa} / \mathrm{m}$ and $\beta_{b n}=$ which can only capture the linear stress-strain relationship of material, the modified PBM can describe the tensile stress developed over the increment of axial strain more accurately. A slight deviation between the results obtained from the experiment and numerical method using the modified PBM, and the breakage of fibre at a strain of $9.5 \%$ indicates the success in 
applying the modified PBM to model coir fibre.

As well as the tension test, a bending test was carried out on coir fibres to determine the shear stiffness $k_{b s}$ of the fibre particle bond. A coir fibre was placed horizontally with one end fixed, while the other end of the fibre was subjected to a vertical load of $0.0545 \mathrm{~g}$ (Fig. 5a). The fibre was bent under the vertical load until it reached a stable condition with a certain deformation. This displacement of fibre was recorded accordingly. The properties of fibre, including its length, diameter, and density were determined; the fibre used in the bending test was approximately $0.3 \mathrm{~mm}$ in diameter and $43.2 \mathrm{~mm}$ long.

Fig. $5 \mathrm{~b}$ shows the cross-section of the fibre while Fig. $5 \mathrm{c}$ shows its bending behaviour as simulated by the modified PBM incorporated in DEM under a vertical load of the same magnitude as applied in the laboratory. In this simulation, 145 particles with a diameter of 0.3 $\mathrm{mm}$ were generated and bonded. With regard to the normal stiffness $k_{b n}$ and the coefficient $\beta_{b n}$ determined from the tension test above, the shear stiffness $k_{b s}$ of $6.1 \times 10^{12} \mathrm{~Pa} / \mathrm{m}$ and $\beta_{b s}$ of 1.0 matched the experimental results quite well. The bending behaviour of fibre modelled by the DEM (Fig. 5c) was clearly the same as the tested coir fibre in the laboratory (Fig. 5a), where the tip of the fibre was stabilised at a vertical displacement of $17 \mathrm{~mm}$.

\section{Structural characteristics of fibre drain}

As described in the experiment determining the hydraulic conductivity of fibres, structural information of fibre drains, including the size and position of individual fibres, was obtained after the hydraulic test. Note that in this study, the fluid-fibre media were mainly investigated with respect to laminar fluid flow that was assumed not to cause a significant disturbance to the fibre structure. The discharge velocity was not determined until the fluid flow became steady. Based on these parameters, fibre drains could be reconstructed numerically in 3D with the same porous features as those tested in the laboratory. The parameters used for bonding fibre particles that were gained from the calibration process (i.e., 
$k_{b n}=1.01 \times 10^{11} \mathrm{~Pa} / \mathrm{m}, \beta_{b n}=0.6$; and $\left.k_{b s}=6.1 \times 10^{12} \mathrm{~Pa} / \mathrm{m}, \beta_{b s}=1\right)$ were applied to these fibres. investigated. Fig. 6a shows a typical cross-section of a dense fibre bundle with a porosity of

$300 \quad 0.35$, where 160 fibres are distributed randomly within a $4 \mathrm{~mm}$ diameter tube. The diameters

301 of the fibre in this case ranges from 112 to $420 \mu \mathrm{m}$, with an average diameter of $235 \mu \mathrm{m}$, but note that due to the large number of fibres packed inside the tube, their locations are relatively uniform. With respect to the coarse-grid approximation method which requires the minimum size of fluid cells be greater than the diameter of particles in this study (O'Sullivan 2011), the porosity of dense fibres were more homogeneous over the fluid cells than the looser ones where fibres have more space to position themselves. Fig. $6 \mathrm{~b}$ shows how DEM could capture the position of fibres within the tube accurately, to ensure a similar porous structure between the DEM and reality. The position of individual fibres in the whole fibre package could be determined exactly on the basis of micro-analyses and this information was then incorporated into the numerical framework.

The segments of fibre drains for twisted and non-twisted bundles with different

312 magnitudes of fibre fraction built in DEM are shown in Fig. 7. All these fibre bundles have 313 the same scale (i.e., cylindrical domain with a diameter of $4 \mathrm{~mm}$ ), and the fibre varies in 314 diameter from 110 to $460 \mu \mathrm{m}$. Fig. 7 a, b, and c represent the loose, medium, and dense 315 bundles where fibres are arranged randomly without twisting, while in Fig. 7 d, e and f, fibres 316 with different porosity are twisted with an approximate angle of $20^{\circ}$. In a non-twisted format, 317 individual fibres are kept almost straight that creates a porous structure consisting of parallel 318 channels, whereas twisted fibres have more complex porous characteristics with longer and 319 more tortuous fluid paths.

Fibres in geoengineering applications might vary from non-twisted to highly twisted, 321 depending on their individual roles. Twisted bundles where the fibres are tightened closely 
together are usually denser and more robust, but less permeable than non-twisted ones. For example, coir fibres that are used for the cores of natural fibre drains are usually twisted highly, whereas jute fibres mainly used for filtering and surface draining are usually not twisted as much. Therefore the hydraulic behaviour of a fibre drain should be considered in close conjunction with the type of fibrous structure and their practical applications.

\section{Modelling fluid flow through fibrous media}

A fluid draining through fibrous media established in DEM was generated in CFD such that the fluid domain was a cylinder, i.e., same as the internal diameter of the tube used for the laboratory tests. Fluid flowed from one end of the domain to the other under the pressure difference between the inlet and the outlet. This pressure difference was created with respect to the hydraulic heads applied in the laboratory. Note that only laminar flow with a hydraulic gradient of less than or equal to 1 was considered in this numerical approach. The fluid velocity at the outlet was obtained from the numerical computation. With respect to the drop in pressure $\Delta p$ and fluid velocity $U_{f, s}$ at the outlet, the hydraulic conductivity of a fibre drain with a length $L$ can be estimated by:

$$
k=\frac{\rho_{f} g U_{f, s} L}{\Delta p}
$$

The fluid domain was discretised into a number of individual cells with a minimum size that was larger than the biggest diameter of fibre particles deposited in the tube. The porosity in each cell was computed individually and was then used to solve the Navier-Stokes equations. To account for the influence of various porous structures in different fibre drains on fluid behaviour, the fluid domain was discretised exactly the same for all cases investigated. Fig. 8a demonstrates how the cylindrical fluid domain is meshed with respect to the largest diameter of fibre particles of $460 \mu \mathrm{m}$. 
In the CFD framework, the critical time step $\Delta t_{f}$ was given by:

$$
\Delta t_{f, m}=\frac{\Delta x}{\left|U_{f}\right|}
$$

where $\Delta x$ is the size of the fluid cell in the direction of fluid flow.

In this study, $\Delta t_{f}=1 \times 10^{-6}$ was used with the exchange between the CFD and DEM

carried out in every 100 time steps $\Delta t_{p}$.

To account for the friction in the tube that could affect how the fluid would behave, a no-slip boundary condition was applied onto the walls of fluid domain. Apart from the path that the fluid would take through the fibre domain, fluid paths were extended at the inlet and outlet to ensure there would be no effect of boundary condition and fluid flow would be fully developed.

Fig. 8 represents a typical fibre drain built in DEM subjected to a fluid flowing along its longitudinal axis. Fluid pressure decreases from the inlet to the outlet over the fibre domain under a hydraulic gradient of 1 . The distribution of fluid velocity and fibre size in a cross-section, extracted from the fibre-fluid system, is shown in Fig. 8b which indicates that the fluid velocity is zero at the boundary (no-slip boundary) and varies according to how the fibre is distributed.

\section{Results and Discussion}

\section{Experimental results}

It it is well known that the hydraulic behaviour of a porous material should be considered with respect to the hydraulic gradient of fluid flow. Fig. 9 shows how the discharge velocity of fluid flowing through a coir fibre drain varies with the measured hydraulic gradients. According to Darcy's law and for a small range of hydraulic gradients, 
365 fluid has laminar flow and there is a linear relationship between the superficial velocity and 366 hydraulic gradient $\left(U_{f, s}=k \times i\right)$. An increasing hydraulic gradient generates a more dynamic 367 portion to the flow (turbulent flow zone) making the relationship between the discharge 368 velocity and hydraulic gradient non-linear. With the coir fibrous media used in this study, the 369 experimental results (Fig. 9) indicate the laminar flow zone generated when the hydraulic 370 gradient is less than 1 . When the hydraulic gradient is more than 1 , fluid flow turns into a transition zone with an increasing portion of turbulent flow. Other studies (Akagi 1994; Bergado et al. 1996; Rawes 1997) have recommended that the largest hydraulic gradient needed to maintain laminar flow in discharge capacity tests of conventional drains should be between 0.1 and 1 . However, it is important to remember that these suggested values might vary depending on the features of the testing models such as scale, confining pressure, and drain characteristics.

There is an obvious relationship between the porosity and hydraulic conductivity of porous media, as confirmed in a number of previous studies (Carman 1937; Sullivan 1942). Fig. 10a shows how the hydraulic conductivity $(k)$ of coir fibres depends on the porosity $(n)$ observed in this study, such that as $n$ decreases from 0.85 to 0.33 , hydraulic conductivity apparently decreases and this reduction steepens when $n<0.5$.

Fig. 10a also indicates a clear difference between the hydraulic behaviour of twisted and non-twisted fibre bundles; where $n>0.65$, these two fibre structures show a slight difference in hydraulic conductivity, but as the fibre becomes denser, there is a clear deviation between the two curves. Twisted bundles have a more complex porous structure than non-twisted ones where individual fibres are almost straight (parallel channels of

387 porosity), the permeability is hence lower. With very dense fibres i.e., $n=0.35$, the hydraulic conductivity of a twisted fibre drain decreases to approximately $1 \times 10^{-5} \mathrm{~m} / \mathrm{s}$, but it is around $6.5 \times 10^{-5} \mathrm{~m} / \mathrm{s}$ for non-twisted types. The denser the fibres being created, the bigger the gap 
390 between the two curves of hydraulic conductivity. This experimental result indicates that the

391 hydraulic behaviour depends on the porous characteristics of fibre drains, and suggests that

392 this relationship should be considered when designing the discharge capacity of fibre drains

393 installed for soft soil improvement.

394 Analytical results

With reference to the $\mathrm{KC}$ equation, hydraulic conductivity calculated over the porosity is shown in Fig. 10b, and compared with the experimental results. In this investigation the properties of fibre drains, including the average fibre diameters and porosities obtained experimentally were adopted (Table 2). The fibres were assumed to be cylindrical with an equivalent diameter computed based on the constant cross-sectional area.

As Fig. 10b shows, for fibre drains with $n>0.6$ (medium to loose fibres), the KC analytical method captures the hydraulic conductivity of the media for twisted and nontwisted forms quite well. For this range of porosity, the biggest deviation between the analytical and experimental results is about $25 \%$ and this is usually acceptable when using the KC approach to predict the permeability of fibrous materials (Gutowski et al. 1987). Note that this accuracy only remained within a narrow range of porosity i.e., $n>0.6$. Since the fibre fraction is increasing, the analytical curves deviate significantly from the experimental ones, while for the dense fibre systems with $n<0.5$, the analytical method results in a far greater difference in hydraulic conductivity compared to the laboratory data. The hydraulic trend predicted by the analytical method for dense fibres deviates completely from the trend observed experimentally, especially for the twisted fibres.

In this analysis, the Kozeny constants $k_{k}$ of 10 and 13.5 were used for non-twisted and

412 twisted fibres, respectively, and these were acceptable at estimating the permeability of

413 medium and loose fibres. However, compared to previous studies (Sullivan 1942; Sherony 414 and Kintner 1971) who generally suggested $k_{k}$ less than 10 for fibrous beds, these values are 
415 larger because of the differences in the porous characteristics of fibres used in different

416 studies. While previous studies used fibres with a parallel arrangement in hydraulic tests, this

417 study created fibre bundles with more complex but realistic structures (non-twisted versus

418 twisted fibres). Moreover, the Kozeny constant was influenced by the specific surface area

419 and the range of porosity, which actually depends directly on the shape and size of the fibres

420 (Li and Gu 2005; Ozgumus et al. 2014). Previous studies used much smaller fibres, e.g., with

421 an equivalent diameter of $7.6 \mu \mathrm{m}$ (Sullivan 1942) and $10 \mu \mathrm{m}$ (Sherony and Kintner 1971),

422 whereas in this study the average diameter of coir fibre was $236 \mu \mathrm{m}$. Li and Gu (2005) also

423 found a relatively high value of $k_{k}$ i.e., 12.75 when they used fibres having a diameter of 170

$424 \mu \mathrm{m}$ in their experimental study. Although the wall-fluid friction was small due to a smooth

425 internal surface of the tube wall adopted, it has not been considered in this investigation.

\section{Numerical results}

427

Fig. 11 represents variations in fluid velocity at specific locations $\left(r_{o}=0.8,1.2\right.$ and

$1.6 \mathrm{~mm}$ ) along the axis of $100 \mathrm{~mm}$ long drain simulated by the coupled CFD-DEM method.

The porosity varies along the drain length (Fig. 11a), resulting in a corresponding variation of

fluid velocity (Fig. 11b). However, the fluid velocity is smaller near the boundary (e.g., $r_{o}=$ $1.6 \mathrm{~mm}$ ) at which the no-slip condition is imposed, although the porosity at this location is higher. These observations are in agreement with a past study by Chen and Papathanasiou (2007_ENREF_8), where it was concluded that non-uniform void distribution in inhomogeneous fibrous structure is expected to give varied flow velocity in the medium. experiments, as shown in Fig. 12. Particularly, the hydraulic conductivity of loose and medium fibres with $n>0.55$ is captured well by the numerical method. An insignificant deviation in hydraulic conductivity obtained from the numerical and experimental methods 
440 between these curves does expand when the fibres are denser. In fact when the porosity falls

441 below 0.4, the differences between the numerical and experimental approaches are more 442 apparent, particularly in twisted fibres.

443 The CFD-DEM coupling technique used in this study was successful at capturing how

444 porous structure could influence on hydraulic behaviour. Fig. 12 shows a deviation in the 445 hydraulic conductivity of the non-twisted and twisted drains modelled by the numerical 446 method that matches the experimental results. For the same discretisation of the fluid domain, 447 different fibre structures with unequal porous distribution result in a variation of parameters 448 such as the porosity $(n)$ and fluid velocity $\left(U_{f}\right)$. Fig. 13 shows how the numerical approach 449 can capture the deviation in fluid flowing through different porous structures of fibrous media 450 (non-twisted and twisted fibres). In Fig. 13, arrows represent fluid paths with their different 451 colours showing the variation of fluid velocity through micro-porous media. Compared to 452 Fig. 13a where fluid flow occurs parallel to the fibre direction, the fluid flow occurs along 453 more tortuous channels in the twisted fibres in Fig. 13b. However the gap between the non454 twisted and twisted curves predicted by the numerical approach in the regime of very low 455 porosity was smaller than the one measured experimentally as shown in Fig. 12, which 456 indicates some limitations of the coupling technique in its ability to capture the accurate hydraulic behaviour in very dense media.

The discrepancies discussed above between the computational and laboratorial 459 investigations can be explained by several major issues, including: ( $i$ ) the no-slip boundary 460 used in the fluid dynamics might not describe the frictional interaction between the fluid and 461 tube walls accurately; (ii) using coarse meshing for the fluid domain could limit of its ability 462 to capture fluid distribution due to complex porous structures, resulting in inaccurate 463 hydraulic behaviour predicted by the numerical method in dense fibres. 
465 An investigation using experimental, analytical and numerical approaches on the hydraulic 466 behaviour of longitudinal flow through brown coconut coir fibres was described in this paper.

467 Two basic types of fibre structure, non-twisted and twisted bundles, were considered. Fibrous structures were reconstructed on the numerical framework based on a series of microanalyses which enabled the porous characteristics to be understood better. The results from the numerical solution were validated with the experimental and Kozeny-Carmen (KC) analytical methods, and the following conclusions can be drawn:

472 1. The hydraulic behaviour of fibrous porous materials depends not only on the porosity but 473 also on the void characteristics of the fibrous media. For example, parallel assembly of fibres (non-twisted), for the same porosity, resulted in a greater permeability than the twisted type in which the fibres create a more complex and longer fluid path.

2. The KC analytical approach could predict the permeability of the fibrous system for $n>$ 0.6 , but there were obvious discrepancies when determining the permeability of denser fibres from those measured in the laboratory. With loose and medium fibrous media $(n>$ 0.6), the Kozeny constants $k_{k}$ of 10 and 13.5 used in the $\mathrm{KC}$ solution could be regarded as sufficiently reliable to compute the hydraulic conductivity of non-twisted and twisted fibres, respectively.

3. By introducing the coefficient $\beta_{b}$ to the conventional PBM, the modified PBM used for bonding particles becomes more flexible to capture different forms of fibre stress-strain relationships, so that more types of fibres can be simulated by the numerical approach.

4. The CFD-DEM coupling technique could depict how porous characteristics affected the hydraulic behaviour of fibrous media, particular those with $n>0.4$. It could also capture the various fluid parameters (e.g., fluid velocity) stemming from differences in the microporous structure within the fibre bundle (drain), and was therefore more accurate in 
489 predicting the hydraulic conductivity than the conventional $\mathrm{KC}$ method which relies $490 \quad$ heavily on the empirical constant $k_{k}$.

\section{Acknowledgements}

492

The authors acknowledge the Australia Research Council for funding this research.

493 Thanks to the National Jute Board of India for providing coir fibres. The $1^{\text {st }}$ author's $\mathrm{PhD}$

494 scholarship is sponsored by the Australia Endeavour Scheme. 


\section{REFERENCES}

Akagi, T. 1994. Hydraulic application of geosynthetics to filtration and drainage problemwith special reference to prefabricated band-shaped drains. In Fifth International Conference on Geotextiles and Geomembranes and Related Products, Singapore. pp. 99-110.

Ali, M. 2010. Coconut fibre- A versatile material and its applications in engineering. In Second International Conference on Sustainable Construction Materials and Technologies, Ancona, Italia. pp. 1441-1454.

Asha, B.S. and Mandal, J.N. 2012. Absorption and discharge capacity tests on natural prefabricated vertical drains. Geosynthetics International, 19: 263-271.

ASTM. 2008. Standard test method for constant head hydraulic transmissivity (In-plane flow) of geotextiles and geotextile related products. ASTM standard D4716. American Society for Testing and Materials, West Conshohocken, Pa.

Bergado, D.T., Manivannan, R. and Balasubramaniam, A.S. 1996. Proposed criteria for discharge capacity of prefabricated vertical drains. Geotextiles and Geomembranes, 14(9): 481-505.

Biswas, S., Ahsan, Q., Cenna, A., Hasan, M. and Hassan, A. 2013. Physical and mechanical properties of jute, bamboo and coir natural fiber. Fibers and Polymers, 14(10): 17261767.

Carman, P.C. 1937. Fluid flow through granular beds. Chemical Engineering Research and Design, 75, Supplement: S32-S48.

Chen, X. and Papathanasiou, T.D. 2007. Micro-scale modeling of axial flow through unidirectional disordered fiber arrays. Composites Science and Technology, 67(7-8): $1286-1293$.

Cho, N., Martin, C.D. and Sego, D.C. 2007. A clumped particle model for rock. International 
Journal of Rock Mechanics and Mining Sciences, 44(7): 997-1010.

Cundall, P.A. and Strack, O.D.L. 1979. A discrete numerical model for granular assemblies. Géotechnique, 29: 47-65.

Defoirdt, N., Biswas, S., Vriese, L.D., Tran, L.Q.N., Acker, J.V., Ahsan, Q., Gorbatikh, L., Vuure, A.V. and Verpoest, I. 2010. Assessment of the tensile properties of coir, bamboo and jute fibre. Composites Part A, 41: 588-595.

Gebart, B.R. 1992. Permeability of unidirectional reinforcements for RTM. Journal of Composite Materials, 26(8): 1100-1133.

Goniva, C., Kloss, C., Hager, A. and Pirker, S. 2010. An open source CFD-DEM perspectives. In Proceeding of the 5th OpenFOAM Workshop, Gothenburg, Sweden.

Gupta, K.M. 2011. Starch based composites for packing applications. In Handbook of bioplastics and biocomposites engineering applications. Edited by S. Pilla. John Wiley \& Sons, Massachusetts.

Gutowski, T.G., Cai, Z., Bauer, S., Boucher, D., Kingery, J. and Wineman, S. 1987. Consolidation experiment for laminate composites. Journal of Composite Materials, 21(7): 650-699.

Kafui, K.D., Thornton, C. and Adams, M.J. 2002. Discrete particle-continuum fluid modelling of gas-solid fluidised beds. Chemical Engineering Science, 57(13): 23952410.

Kim, J.H. and Cho, S.D. 2009. Pilot scale field test for natural fiber drain. In Geosynthetics in civil and environmental engineering. Edited by G. Li, Chen, Y. \& Tang, X. Springer Berlin Heidelberg. pp. 409-414.

Kim, Y.W., Park, J.Y. and Jang, Y.S. 2001. Consolidation efficiency of natural and plastic geosynthetic band drains. Geosynthetics International, 8(4): 283.

Kloss, C. and Goniva, C. 2010. LIGGGHTS: a new open source discrete element simulation 
software. In Proceeding of the 5th International Conference on Discrete Element Methods, London, UK.

Kloss, C., Goniva, C., Hager, A., Amberger, S. and Pirker, S. 2012. Models, algorithms and validation for opensource dem and cfdem. Progress in Computational Fluid Dynamics, an International Journal, 12(2): 140-152.

Lee, S.L., Karunaratne, G.P., Ramaswamy, S.D., Aziz, M.A. and Das Gupta, N.C. 1994. Natural geosynthetic drain for soil improvement. Geotextiles and Geomembranes, 13(6-7): 457-474.

Li, J. and Gu, Y. 2005. Coalescence of oil-in-water emulsions in fibrous and granular beds. Separation and Purification Technology, 42(1): 1-13.

Lisjak, A. and Grasselli, G. 2014. A review of discrete modeling techniques for fracturing processes in discontinuous rock masses. Journal of Rock Mechanics and Geotechnical Engineering, 6(4): 301-314.

Nguyen, T.T. and Indraratna, B. 2016. Hydraulic behaviour of parallel fibres under longitudinal flow: a numerical treatment. Canadian Geotechnical Journal, 53(7): 1081-1092. doi: http://www.nrcresearchpress.com/doi/abs/10.1139/cgj-20150213\#.VviZaeJ97IU.

O'Sullivan, C. 2011. Particulate discrete element modelling: a geomechanics perspective. Spon Press (an imprint of Taylor \& Francis), London.

Ozgumus, T., Mobedi, M. and Ozkol, U. 2014. Determination of kozeny constant based on porosity and pore to throat size ratio in porous medium with rectangular rods. Engineering Applications of Computational Fluid Mechanics, 8(2): 308-318.

Potyondy, D.O. and Cundall, P.A. 2004. A bonded-particle model for rock. International Journal of Rock Mechanics and Mining Sciences, 41(8): 1329-1364.

Rao, K.M.M. and Rao, K.M. 2007. Extraction and tensile properties of natural fibers: Vakka, 
date and bamboo. Composite Structures, 77(3): 288-295.

Rasband, W.S. 2014. ImageJ. U. S. National Institutes of Health, Bethesda, Maryland, USA, http://imagej.nih.gov/ij/.

Rawes, B.C. 1997. Critical parameters for specification of prefabricated vertical drains. Geosynthetics International, 4: 51-64. doi: 10.1680/gein.4.0088.

Sharma, S. and Siginer, D.A. 2010. Permeability Measurement Methods in Porous Media of Fiber Reinforced Composites. Applied Mechanics Reviews, 63: 02080201-02080219.

Sherony, D.F. and Kintner, R.C. 1971. Coalescence of an emulsion in a fibrous bed: Part I. Theory Canadian Journal of Chemical Engineering, 49(3): 314-320.

Som, N., Chattopadhyay, B.C. and Sanyal, T. 2009. A manual on use of jute geotextiles in civil eingieering, 4th Edition. Jute Manufactures Development Council, Kolkata, India.

Sullivan, R.R. 1942. Specific surface measurements on compact bundles of parallel fibers. Journal of Applied Physics, 13(11): 725-730.

Venkatappa Rao, G., Sampath Kumar, J.P. and Banerjee, P.K. 2000. Characterization of a braided strip drain with coir and jute yarns. Geotextiles and Geomembranes, 18(6): 367-384.

Zhou, Z.Y., Kuang, S.B., Chu, K.W. and Yu, A.B. 2010. Discrete particle simulation of particle-fluid flow: model formulations and their applicability. Journal of Fluid Mechanics, 661: 482-510. doi: doi:10.1017/S002211201000306X.

Zhu, H.P., Zhou, Z.Y., Yang, R.Y. and Yu, A.B. 2007. Discrete particle simulation of particulate systems: theoretical developments. Chemical Engineering Science, 62(13): $3378-3396$. 


\section{TABLES}

\begin{tabular}{|c|c|c|c|c|c|c|}
\hline \multirow{2}{*}{$\begin{array}{l}\text { Density } \\
\left(\mathrm{kg} / \mathrm{m}^{3}\right)\end{array}$} & \multicolumn{2}{|c|}{ Water content $(\%)$} & \multirow{2}{*}{$\begin{array}{l}\text { Tensile } \\
\text { strength } \\
(\mathrm{MPa})\end{array}$} & \multirow{2}{*}{$\begin{array}{c}\text { Elastic } \\
\text { Modulus } \\
(\mathrm{GPa})\end{array}$} & \multirow{2}{*}{$\begin{array}{c}\text { Diameter } \\
(\mathrm{mm})\end{array}$} & \multirow{2}{*}{$\begin{array}{l}\text { Length } \\
(\mathrm{mm})\end{array}$} \\
\hline & Dry & Saturated & & & & \\
\hline 1160 & 14 & 94 & $188 \pm 34$ & 4.2 & $\begin{array}{l}0.102- \\
0.495\end{array}$ & $65-250$ \\
\hline
\end{tabular}

Table 1 Physical properties of brown coir fibres 


\begin{tabular}{|c|c|c|c|c|}
\hline \multirow{2}{*}{ Test } & \multicolumn{2}{|c|}{ Twisted fibres } & \multicolumn{2}{c|}{ Non-Twisted fibres } \\
\cline { 2 - 5 } & Porosity & $\begin{array}{c}\text { Average diameter } \\
D_{f}(\mathrm{~m})\end{array}$ & Porosity & $\begin{array}{c}\text { Average diameter } \\
D_{f}(\mathrm{~m})\end{array}$ \\
\hline 1 & 0.330 & $2.362 \mathrm{E}-004$ & 0.351 & $2.351 \mathrm{E}-004$ \\
\hline 2 & 0.418 & $2.346 \mathrm{E}-004$ & 0.434 & $2.342 \mathrm{E}-004$ \\
\hline 3 & 0.523 & $2.396 \mathrm{E}-004$ & 0.547 & $2.319 \mathrm{E}-004$ \\
\hline 4 & 0.628 & $2.407 \mathrm{E}-004$ & 0.682 & $2.401 \mathrm{E}-004$ \\
\hline 5 & 0.822 & $2.318 \mathrm{E}-004$ & 0.850 & $2.398 \mathrm{E}-004$ \\
\hline
\end{tabular}

Table 2 Parameters for the permeability estimated by the Kozeny-Carmen solution 


\section{FIGURES}

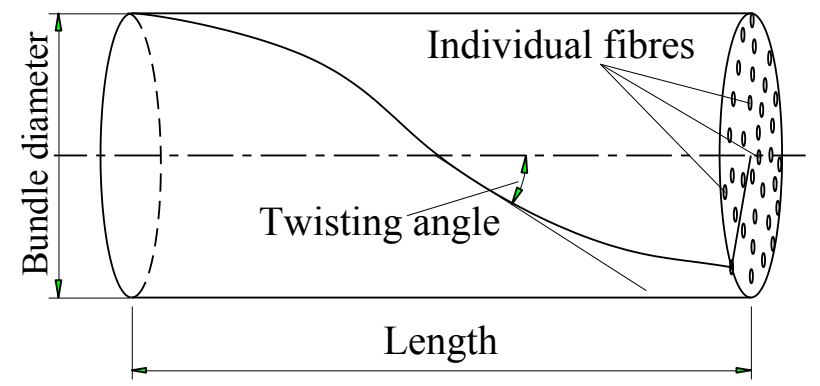

(a)

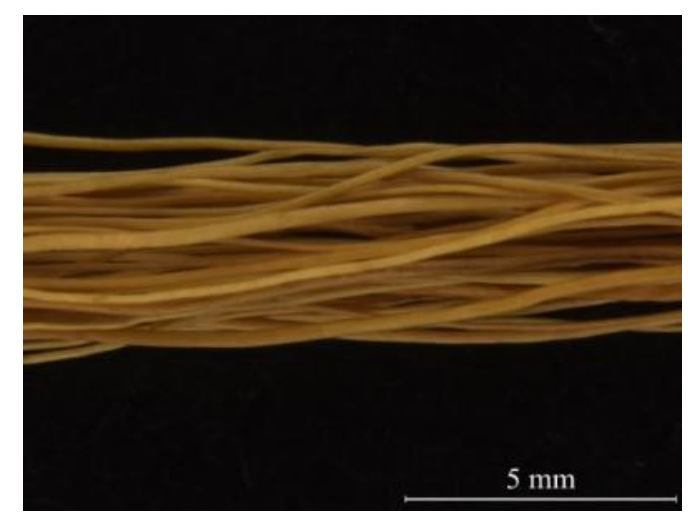

(b)

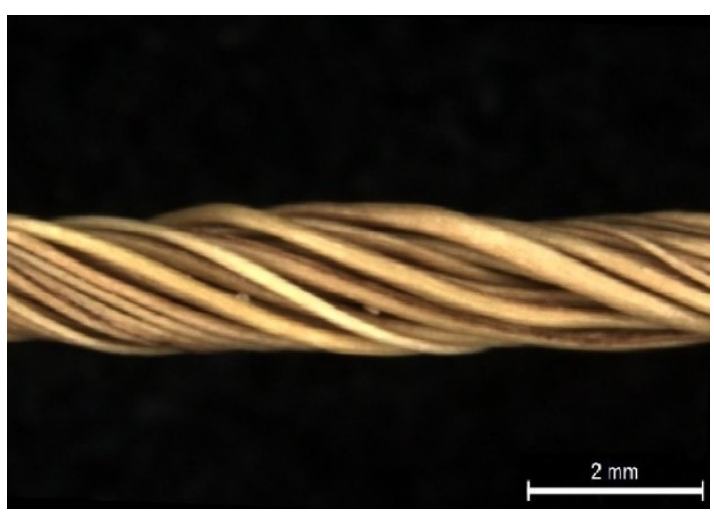

(c)

Fig. 1 Fibre drains composed of coir fibres under optical microscope: (a) Schematic details of twisted fibre bundle; (b) Non-twisted fibres; and (c) Twisted fibres 


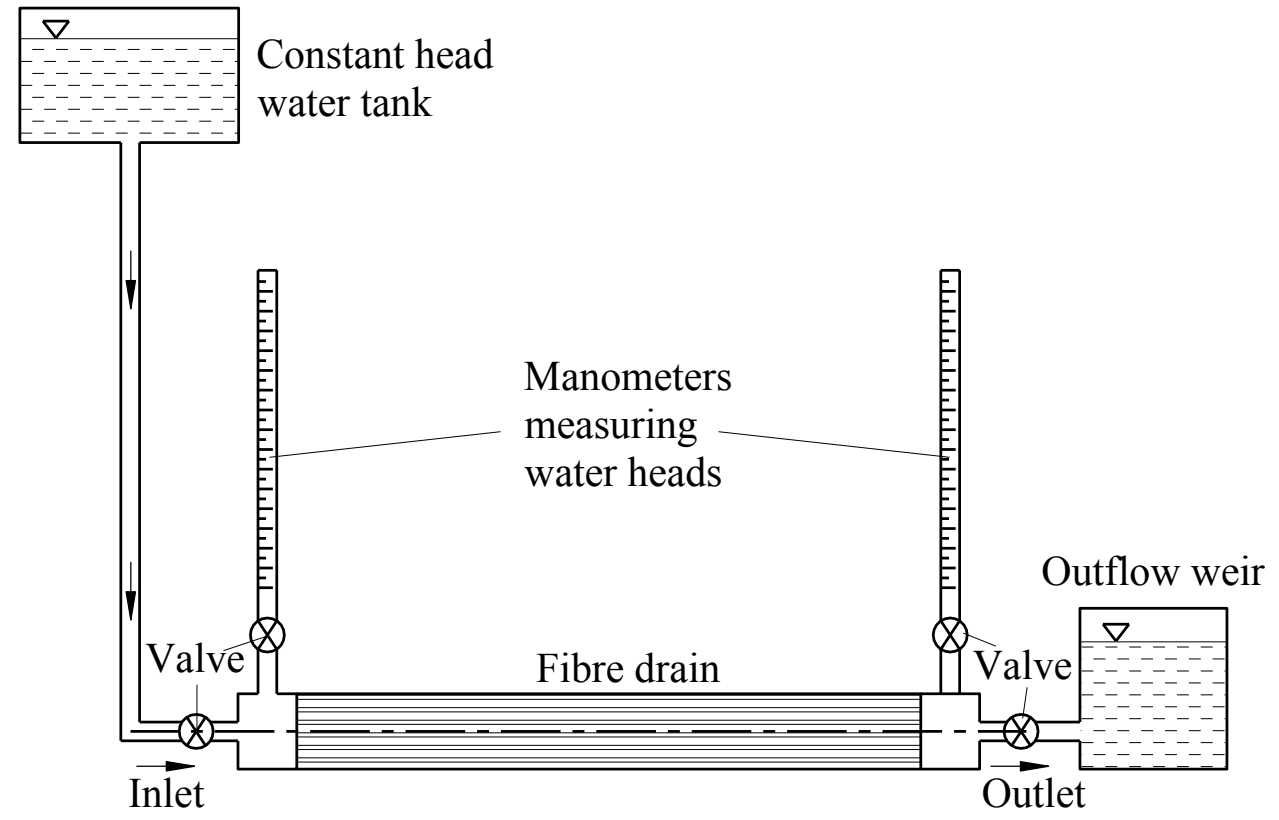

(a)

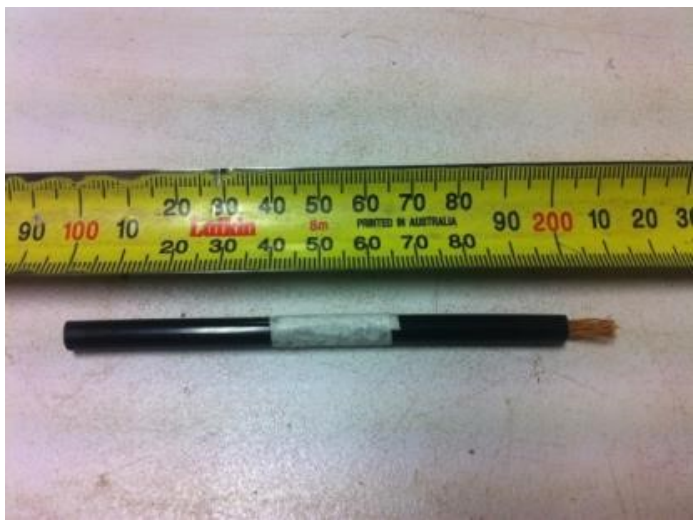

(b)

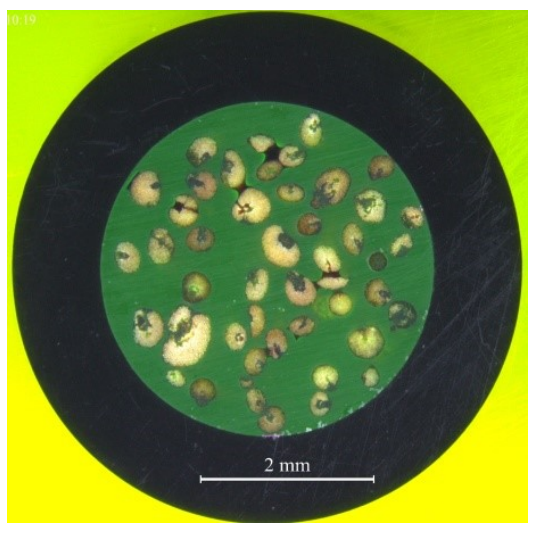

(c)

Fig. 2 Experiment model to determine hydraulic conductivity of fibrous drain: (a) Schematics of experiment model; (b) Fibre tube; (c) Cross-section of fibre tube after casting 


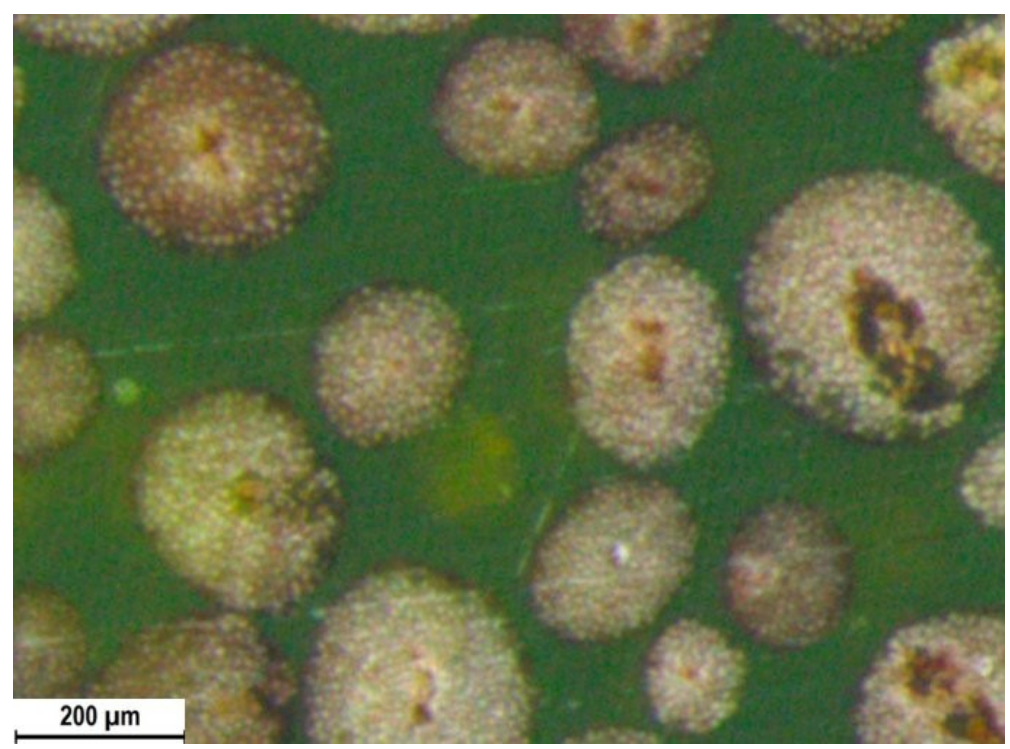

(a)

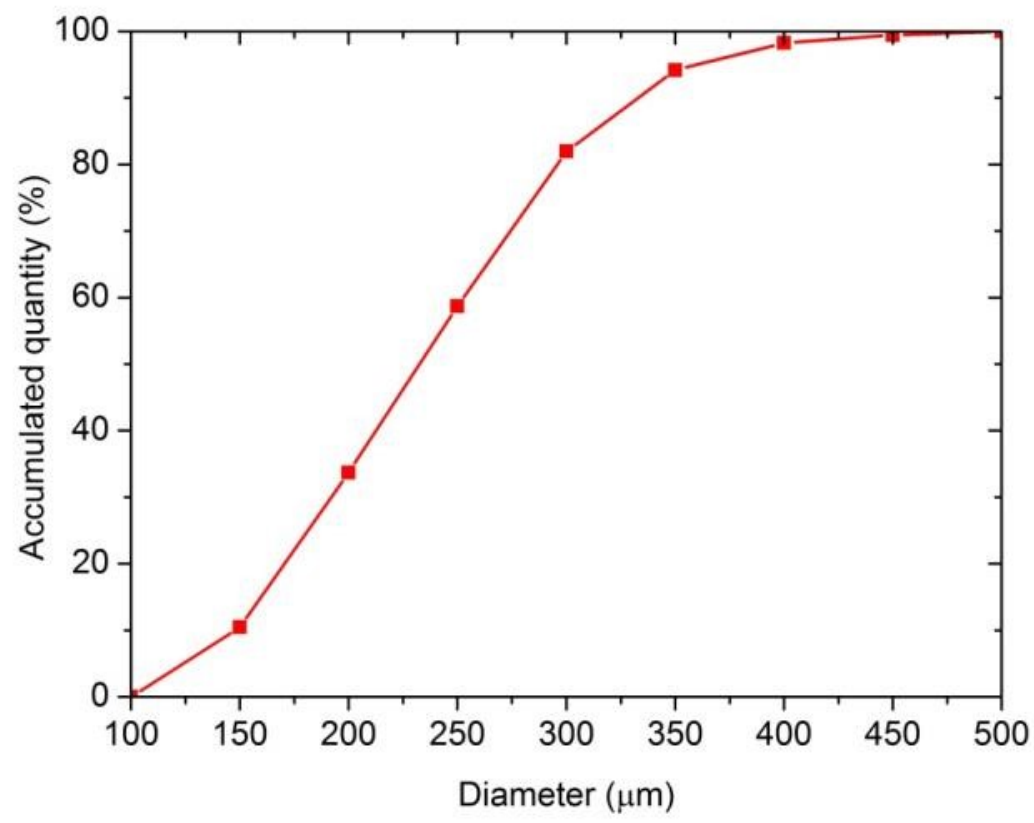

(b)

Fig. 3 Physical properties of coconut coir fibres: (a) Cross-section of the fibres under microscope observation; (b) Size distribution of fibres in a bundle 


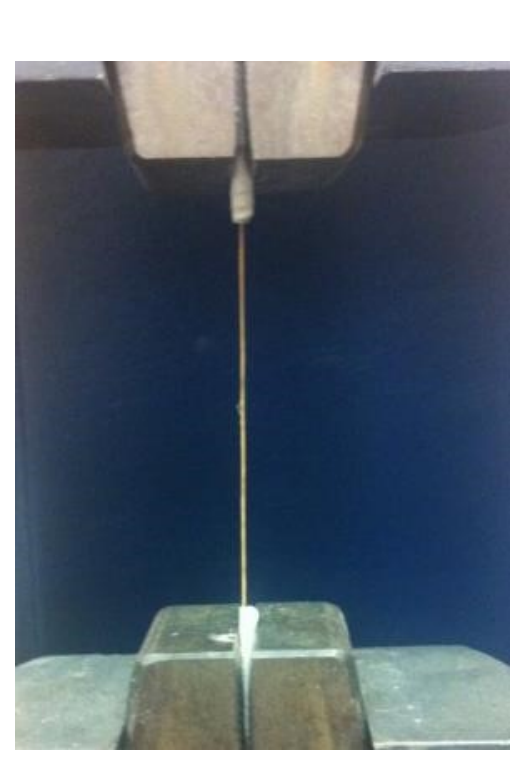

(a)

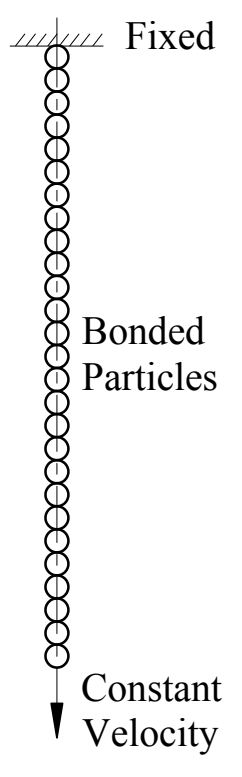

(b)

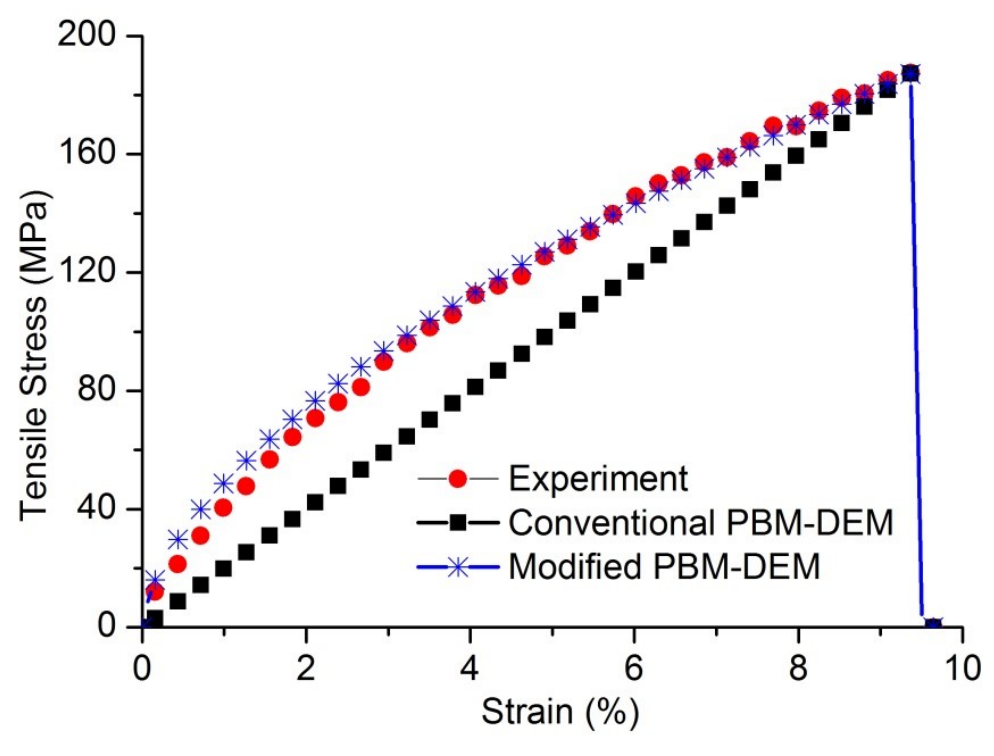

(c)

Fig. 4 Tension test on individual coir fibre: (a) Experiment; (b) DEM modelling; (c) Stressstrain relationship by experiment and DEM modelling 


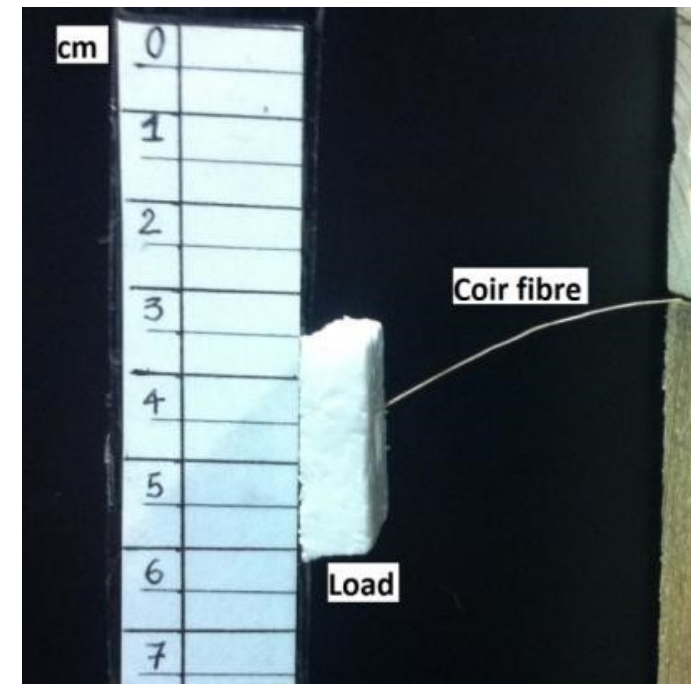

(a)

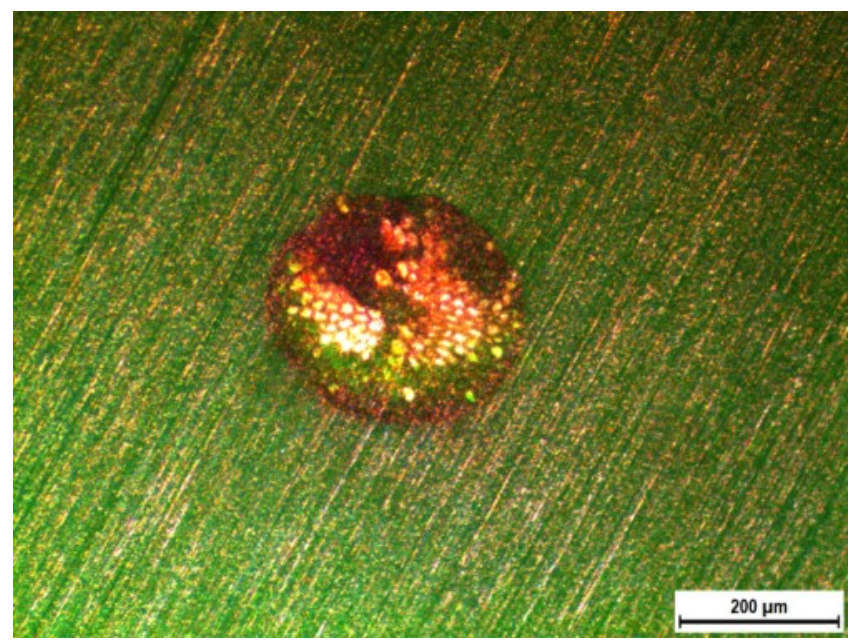

(b)

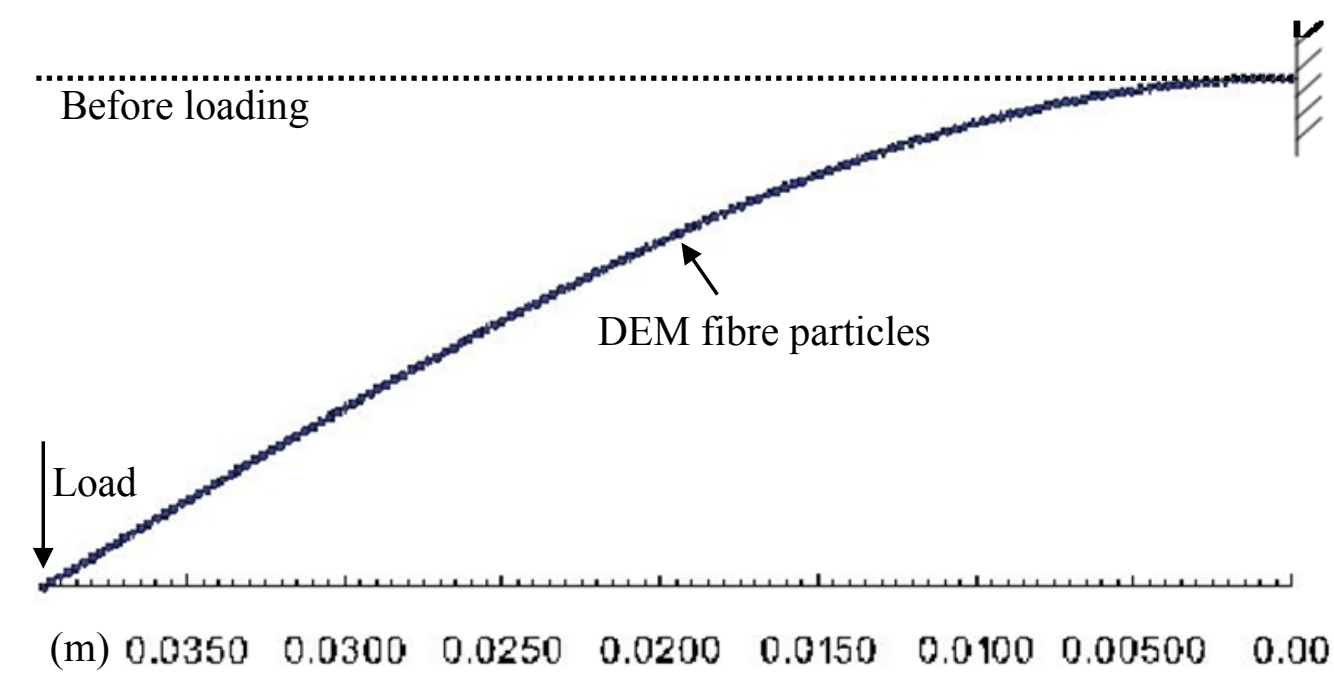

(c)

Fig. 5 Bending test on individual coir fibre: (a) Experimental model; (b) Fibre cross-section under optical microscope observation; (c) DEM modelling 


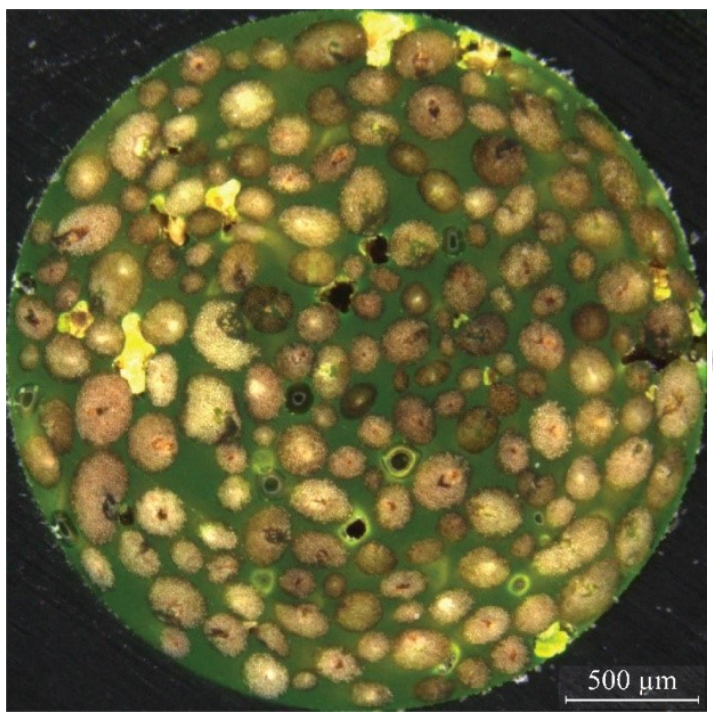

(a)

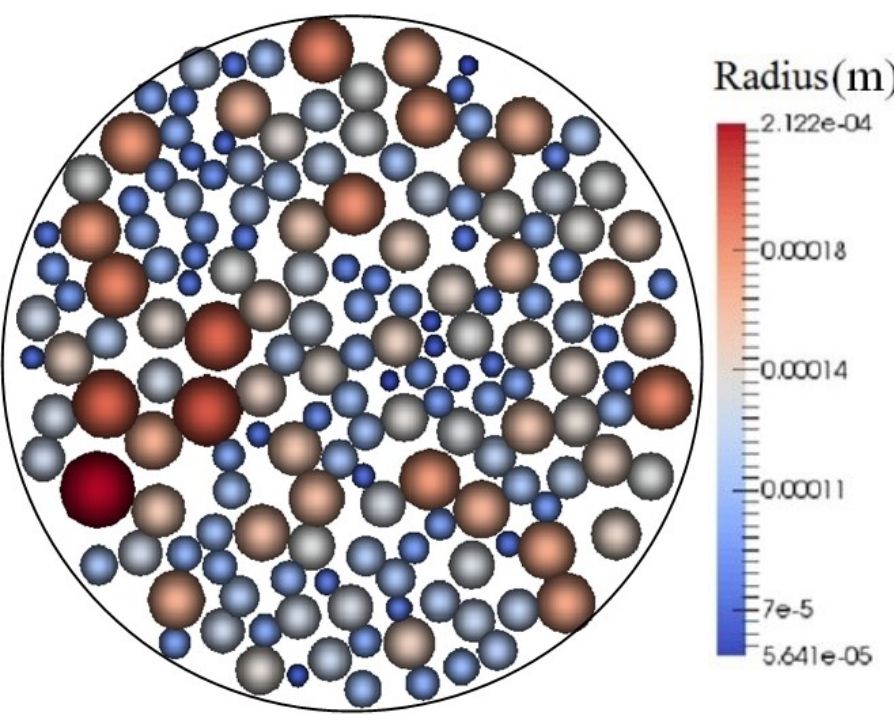

(b)

Fig. 6 Capturing the position of fibre particles in a drain: (a) Cross-section of dense coir fibres under microscope; (b) Fibres modelled in DEM 


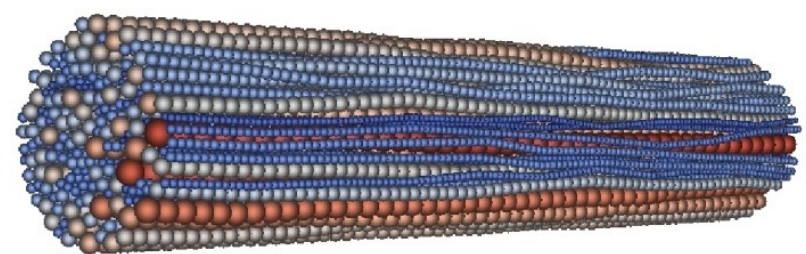

(a) Dense non-twisted fibres, $n=0351$

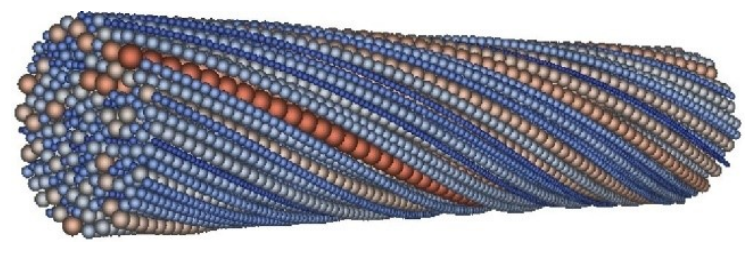

(d) Dense twisted fibres, $n=0.330$

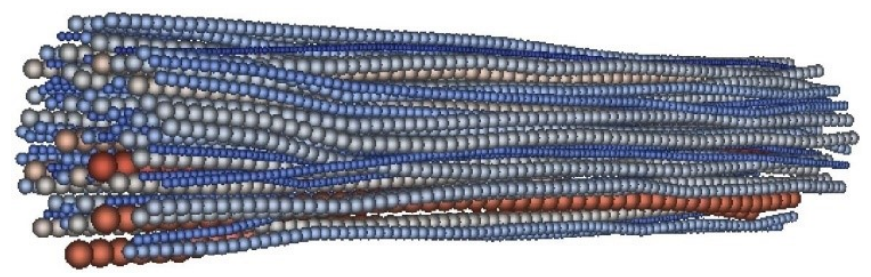

(b) Medium non-twisted fibres, $n=0.682$

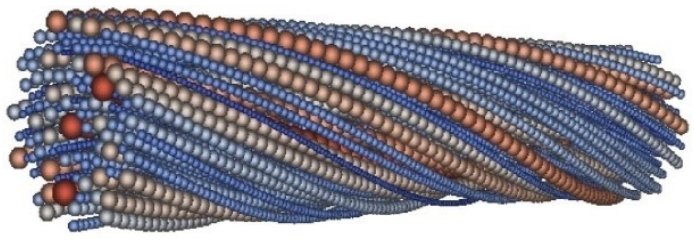

(e) Medium twisted fibres, $n=0.628$

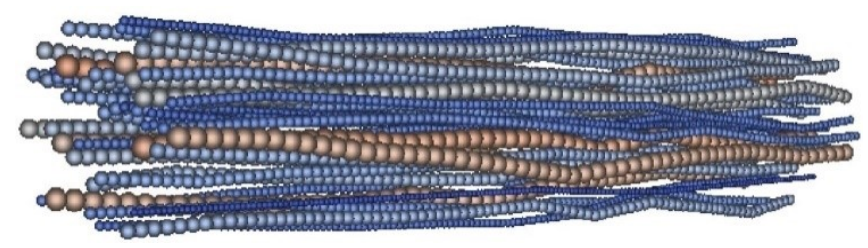

(c) Loose non-twisted fibres, $n=0.850$

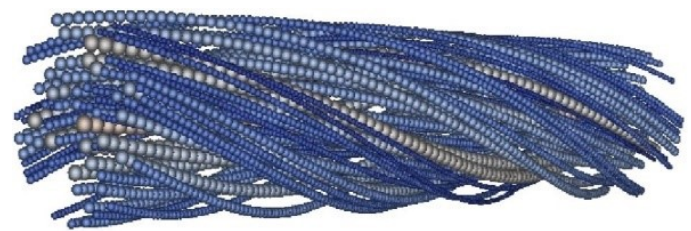

(f) Loose twisted fibres, $n=0.822$

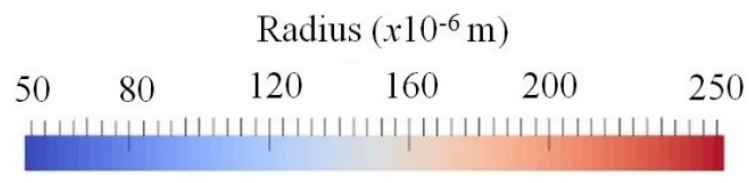

Fig. 7 Segments of fibre bundles built in DEM with different porous structure: twisted and non-twisted fibres 


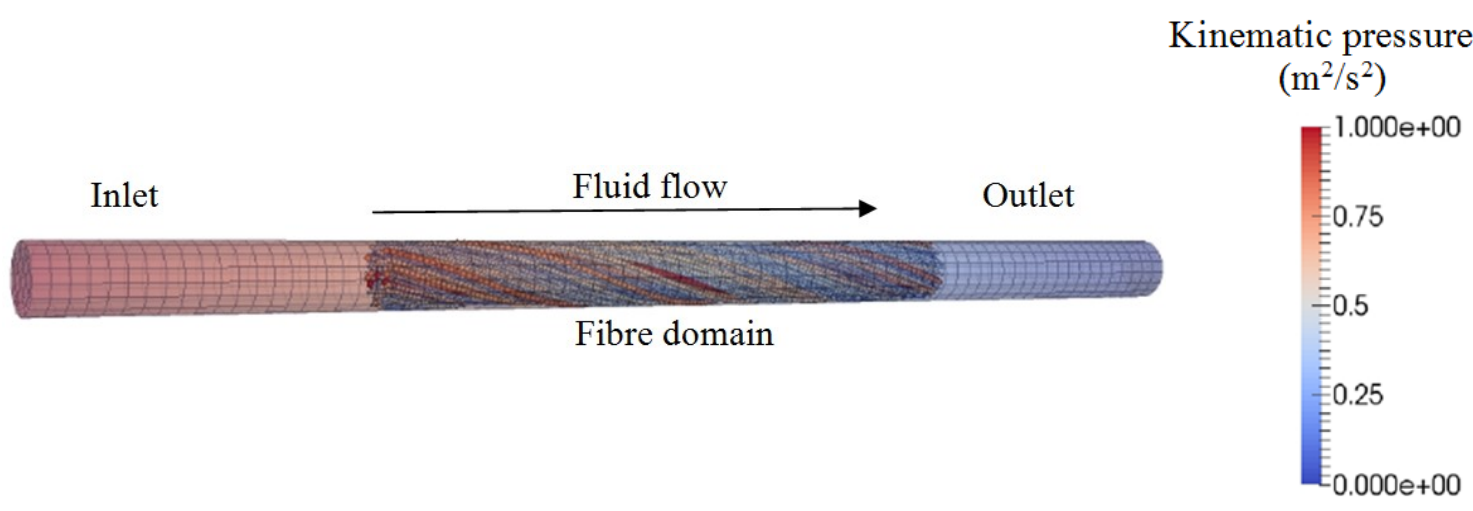

(a)

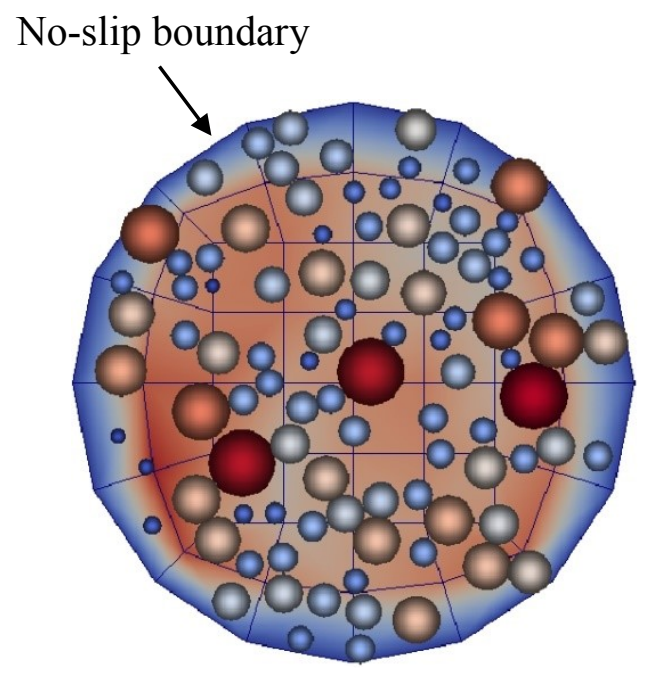

Fluid velocity (m/s) Particle radius (m)
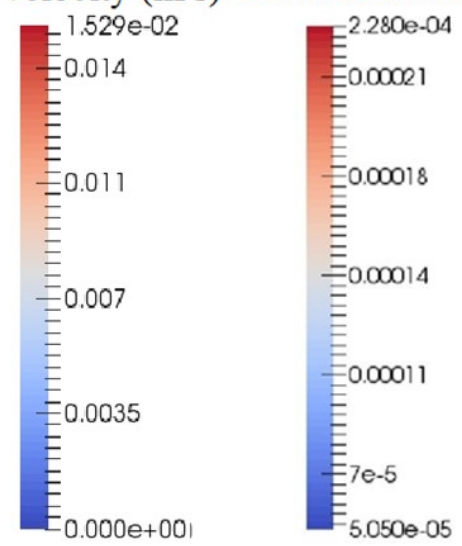

(b)

Fig. 8 Meshing fluid domain: (a) Longitudinal discretization; (b) Cross-section of fluid domain with the fluid velocity distribution in relation to fibre particles 


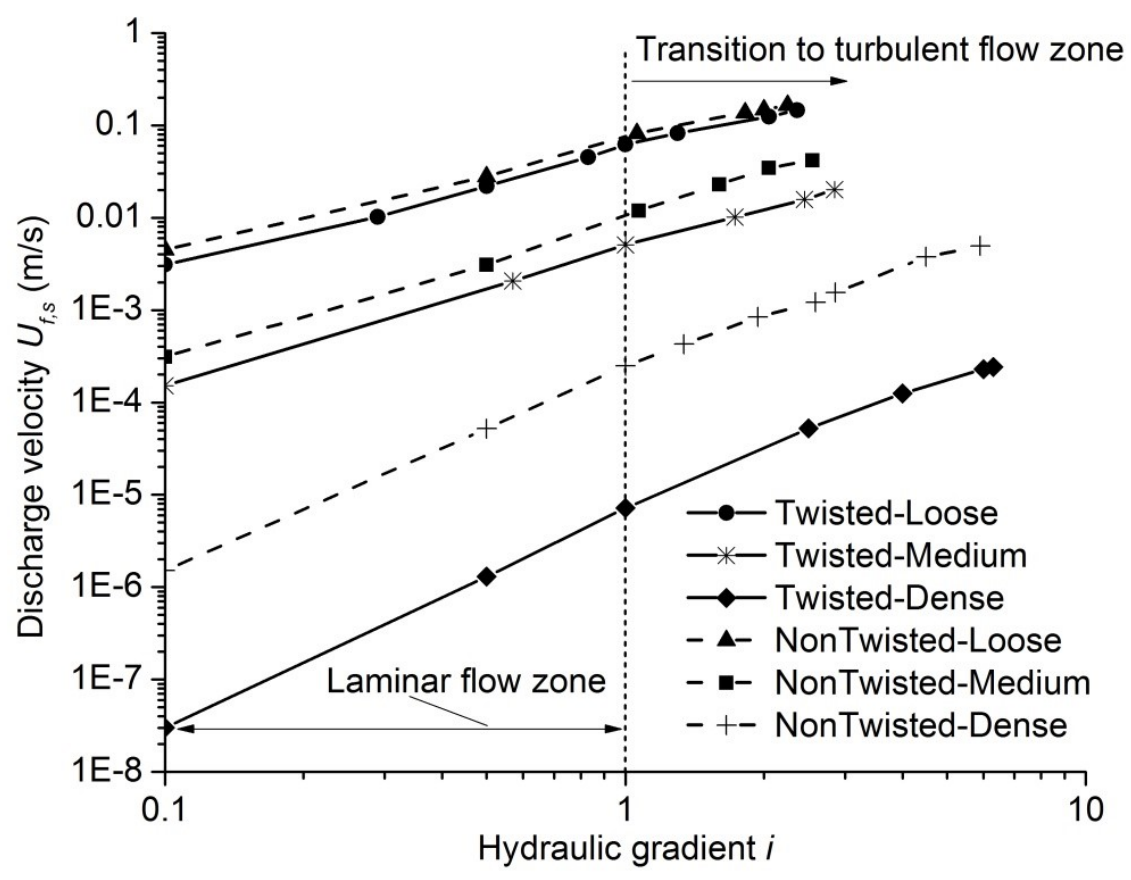

Fig. 9 Experimental discharge velocity of fluid flowing through fibre drain over hydraulic gradient 


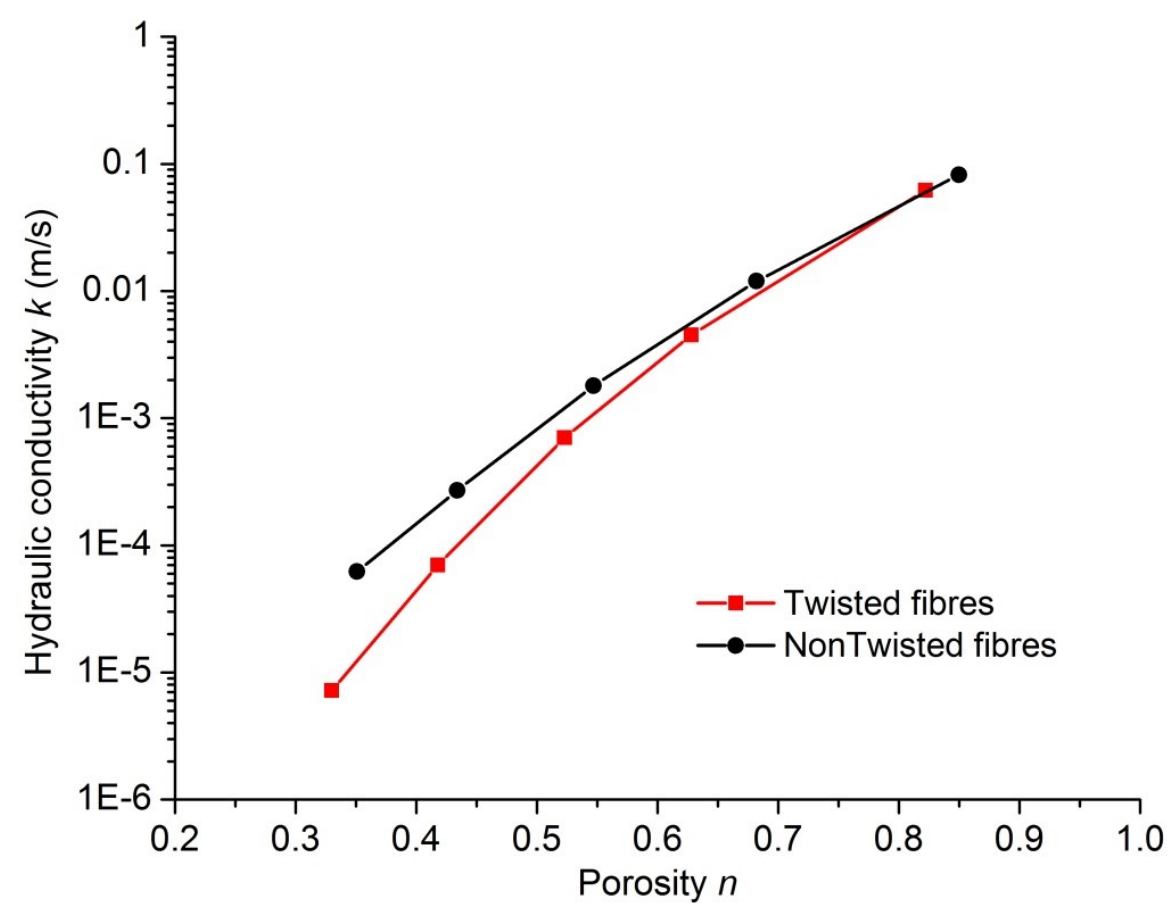

(a)

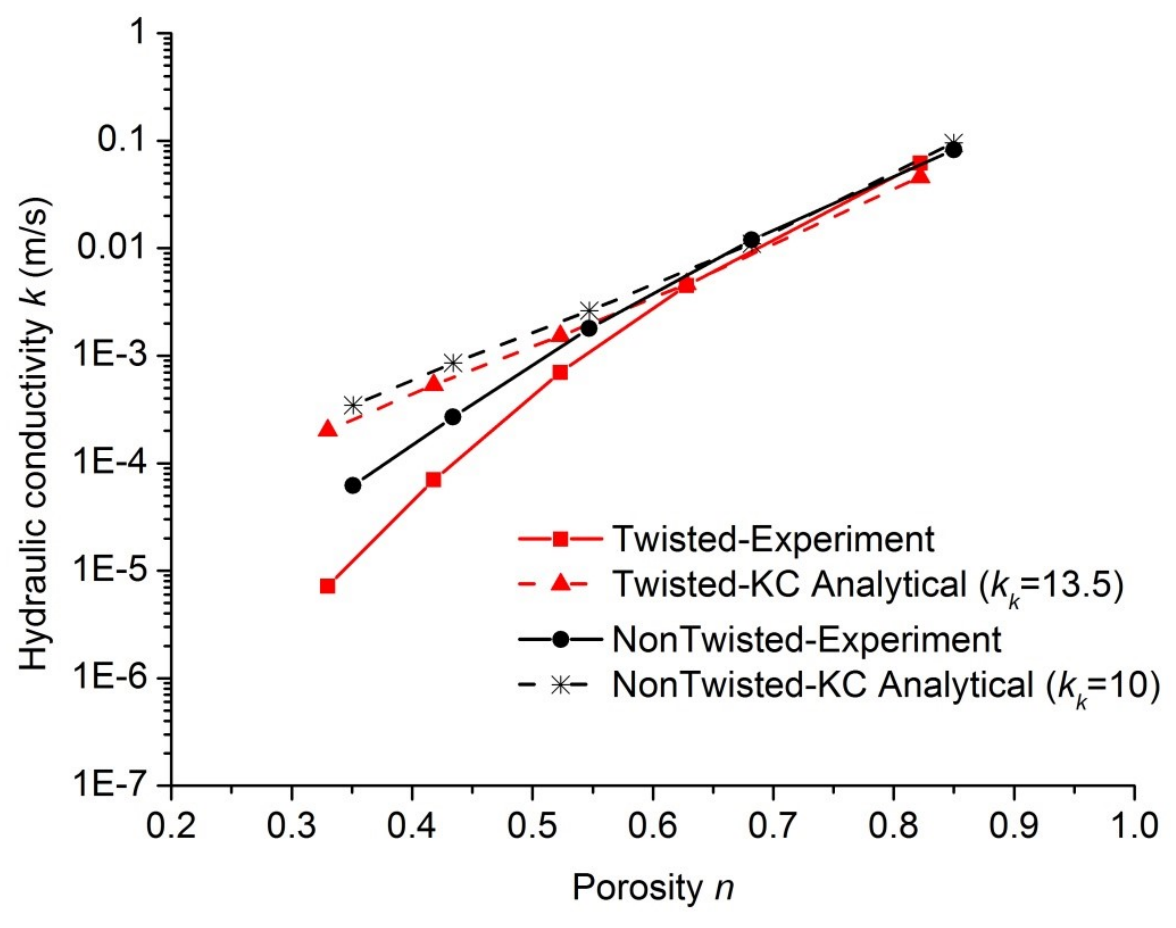

(b)

Fig. 10 Hydraulic conductivity of coir fibres obtained by: (a) Experimental; and (b) In comparison with the K-C analytical methods 

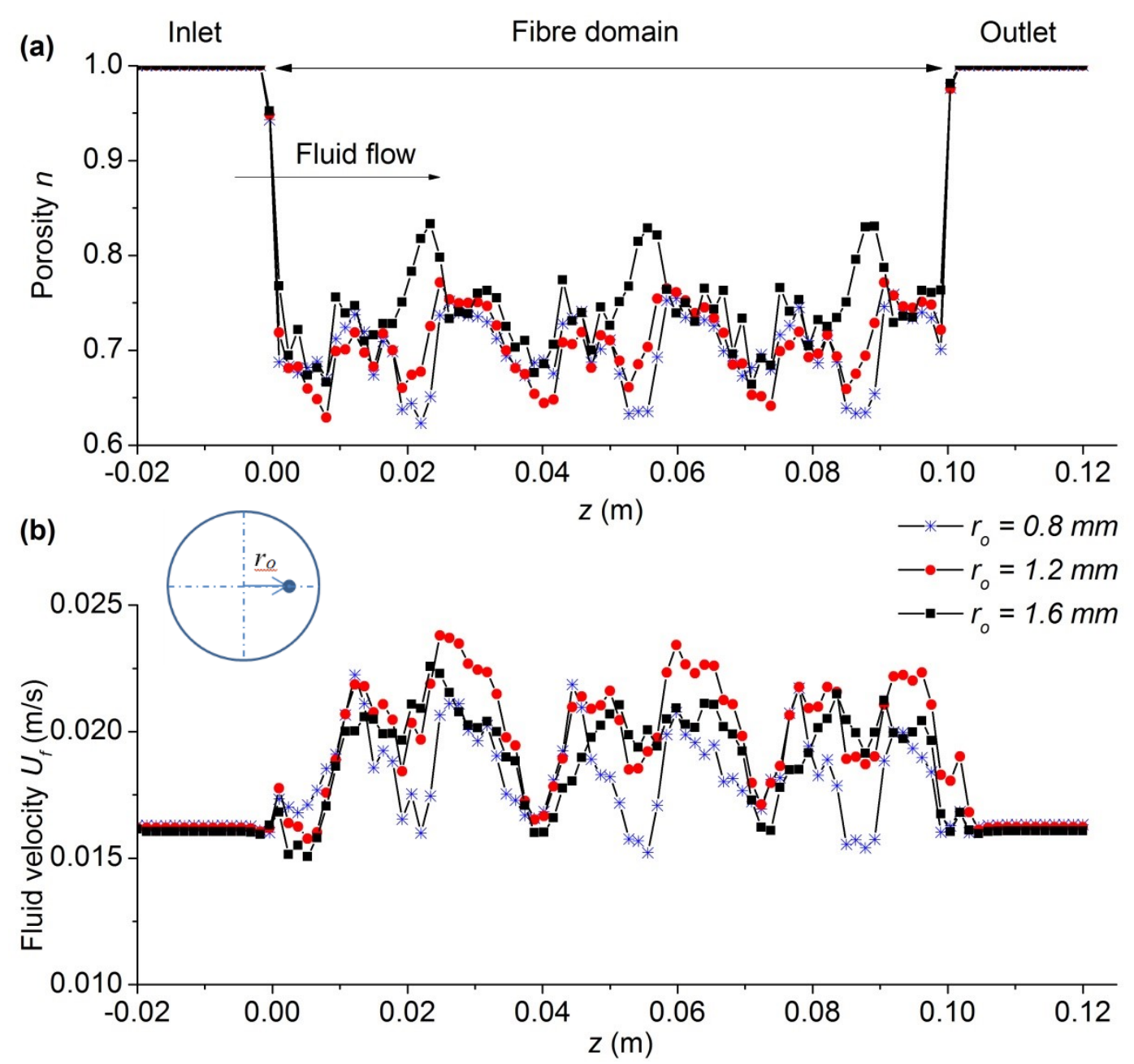

Fig. 11 Variation of parameters over fluid cells: (a) Porosity; and (b) Fluid velocity. 


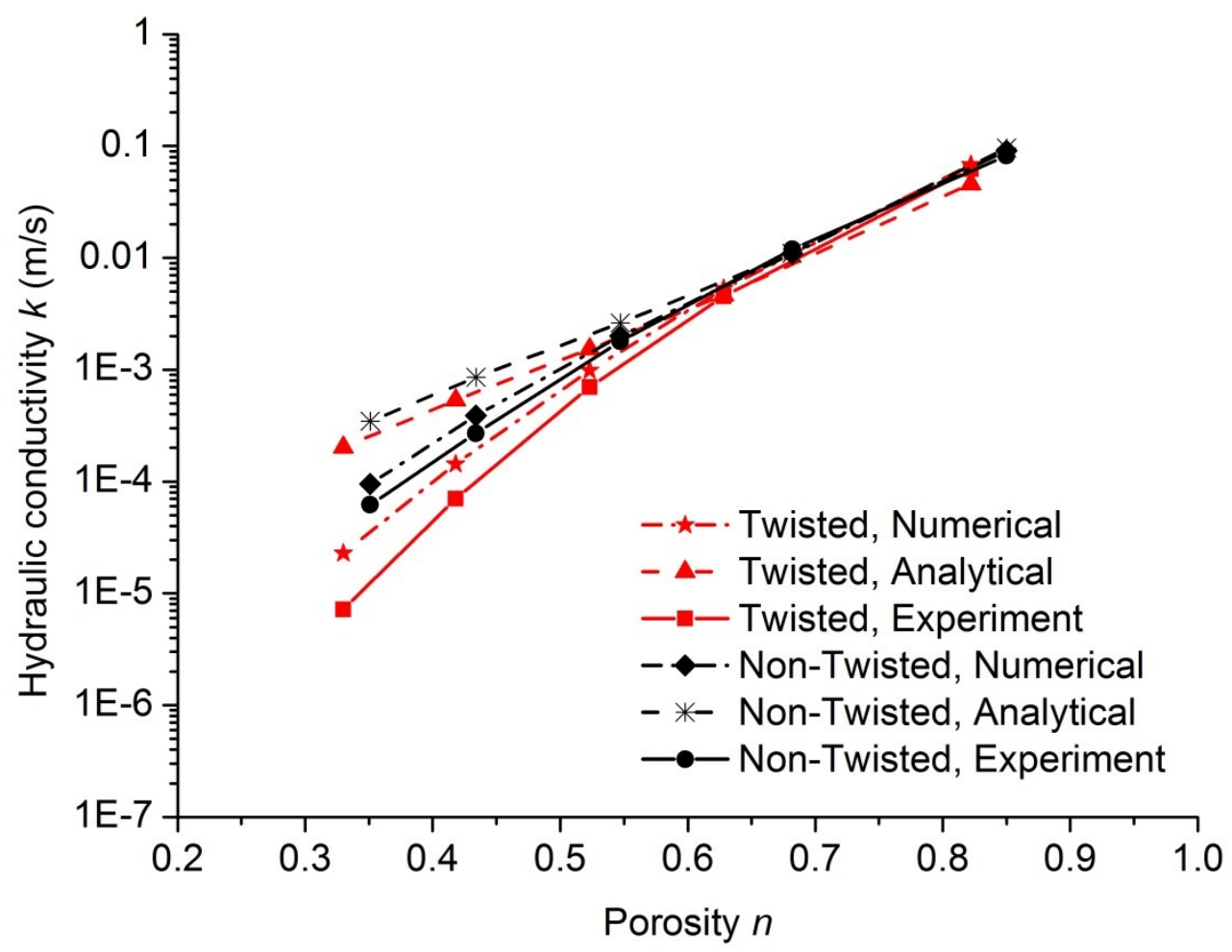

Fig. 12 Hydraulic conductivity predicted by the CFD-DEM approach in comparison with the experimental and analytical methods 


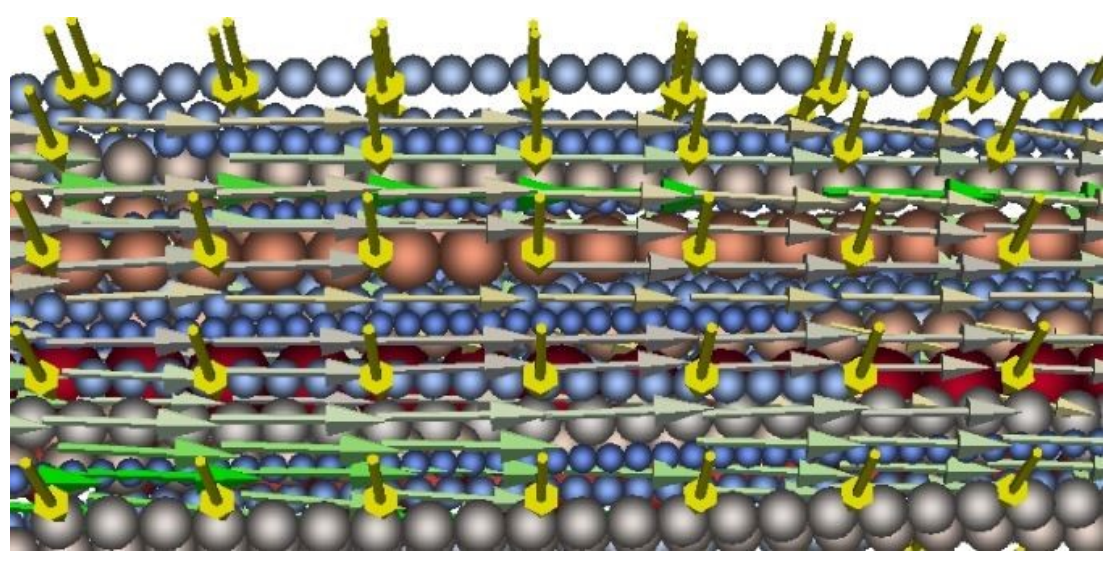

(a)

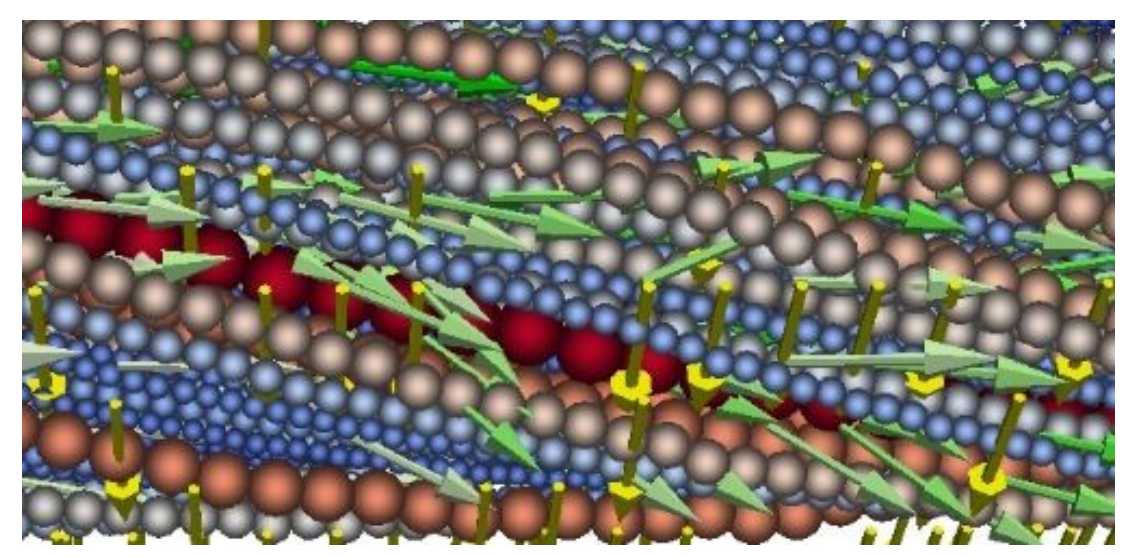

Fluid velocity $U_{f}(\mathrm{~m} / \mathrm{s})$

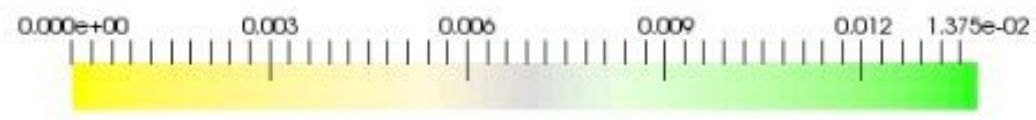

(b)

Fig. 13 Fluid flowing through different fibrous porous structures: (a) Non-twisted; and (b) Twisted fibres; captured by the CFD-DEM method. 